\title{
Drivers of land use change and carbon mapping in the savannah area of Ghana
}

\author{
Addo Koranteng ${ }^{1} \bowtie$, Isaac Adu-Poku ${ }^{2}$,Tomasz Zawila-Niedzwiecki ${ }^{3}$ \\ ${ }^{1}$ Kumasi Technical University, Institute of Research, Innovation and Development, Kumasi, Ghana, \\ e-mail:dr.adokay@gmail.com \\ ${ }^{2}$ Kwame Nkrumah University of Science and Technology, Department of Geomatic Engineering, Kumasi, Ghana \\ ${ }^{3}$ Directorate General of the State Forests, Grójecka 127, 02-124 Warszawa, Poland
}

\section{Abstract}

Land-use and land-cover change in both forest reserves and off-reserves is a critical issue in sub Saharan Africa. Deforestation and conversion of forest land to agricultural land continue to be one of the major environmental problems in Africa, and for that matter, Ghana cannot be exceptional; and its resultant effect is the loss in the ecological integrity and the quality of forests, resulting in carbon loss and the resultant climate change effects (FAO 2016). The study area covers the Community Resource Management Areas (CREMA) of the Mole National Park in Ghana, and this study reveals that the area is well endowed with a diverse composition and structure of woodland including dense, open and riverine stretches, which - under the national definition of forest - qualifies as forest. The results reveal that there had been an annual deforestation rate of $0.11 \%$ over the period of review. It was concluded from the study that woodland had high carbon stocks with an average carbon of $80 \mathrm{tC} / \mathrm{ha}$, the highest being $194 \mathrm{tC} / \mathrm{ha}$ and the lowest being $7 \mathrm{tC} / \mathrm{ha}$, which was recorded in the dense woodland and grassland respectively. The fluxes within the land sector in the study area are moderate and the potential of the area to qualify for as REDD + is very high. However, the drivers of deforestation, especially bush fires and illegal timber harvesting, are challenges that need to be addressed.

\section{KeY WORDS}

deforestation, land use change, carbon mapping, remote sensing, REDD+

\section{INTRODUCTION}

Savannah woodland is estimated to cover approximately two thirds of Ghana's land area and contains $20 \%$ of the nation's population (GSS 2013). About 70\% of Ghana's total supply of firewood and charcoal, estimated at 16 million $\mathrm{m}^{3}$, comes from savannah zones (FPP-PASCOFC Report 2013).
Ghana is endowed with abundant natural resources that have played an important role in national development. As a result of unsustainable exploitation, damage has been caused to productive forest and savannah lands (Antwi et. al. 2017; Ayuk and Oku 2017). The burgeoning population is presently exerting immense pressure on natural resources. The ecological dynamics has also not been spared due to rapid loss of biological diver- 
sity, wildlife populations and adverse effects of climate change (Asante 2014). These anthropogenic effects on the resources is likely to persist considering the expected increase in the demand for land based products driven by human population growth, diet change and consumption of energy (Haberl et al. 2014; Rockstrom et al. 2009).

According to a study conducted by the Forestry Commission of Ghana (FC) under the Forest Preservation Programme (FPP), deforestation and conversion of forest land to agricultural lands continue to be one of the major environmental problems in Ghana. It is considered as a major threat to both on and off forest reserve resources. In the off-reserve areas, it is attributed to the conversion of forest into agricultural use, wildfires, and over-exploitation of timber, wood fuel production and establishment of new settlements or expansion of existing ones among others. The effects of these activities are the loss in the ecological integrity and quality of the forest resulting in carbon loss and the resultant climate change effects (Forestry Commission of Ghana 2014).

Land-cover changes have significantly impacted forest carbon stocks, which has been recognized worldwide as contributing to $\mathrm{CO}_{2}$ emissions into the atmosphere resulting in global warming (Innes 2016). Land-use and land-cover change are responsible for a third of the global anthropogenic Greenhouse Gas (GHG) emissions over the last 150 years (Houghton 2005). On-going deforestation and forest degradation is the major source of current GHG emissions in many tropical developing countries (Van Der Werf et al. 2009).

The Forestry Commission in 2012 under the Forest Preservation Program (FPP) developed land use maps and land use change for a twenty-year period (1990-2010). The project further estimated the carbon content of the various vegetation zones in Ghana with much concentration in the High Forest Zones (HFZ) of the country, as little was done on the savannah woodlands. Consequently, the modelling of the carbon content for the country was based on a pilot area that did not include much of the savannah woodlands. In addition to this, almost all forest inventory projects in the past were concentrated in the high forest zone of Ghana with the exception of the Northern Savannah Biodiversity Project, which established some informa- tion on the savannah woodlands. However, information on the rates of deforestation, forest degradation and forest conversion in the savannah woodland as well as carbon stocks level is insufficient and has serious implication for sustainable management of woodlands forests.

\section{Aims and objectives}

The main aim of this study is to appraise land use and baseline studies of the natural woodland in terms of its spatial coverage and trend of use, and its ability to withstand the incessant cutting in the northern parts of Ghana.

The Objective:

- What are the various land-use and land-cover categories in the study area?

- What are the trajectories of the land-use change conversions and the drivers pushing that change within the area?

- To determine the carbon content within the area.

\section{MATERIALS AND METHODS}

\section{Study Area}

Study area (Fig. 1) falls within the Guinea savannah ecological zone of Ghana and covers approximately 122,240 ha. The dominant vegetation type is open woodland within grass trees mosaic. It lies between latitudes $9^{\circ} 10^{\prime} \mathrm{N}$ and $9^{\circ} 45^{\prime} \mathrm{N}$ and longitudes $1^{\circ} 10^{\prime}$ and $1^{\circ} 50^{\prime} \mathrm{W}$, and stretches from West Gonja to North Gonja Districts of northern Ghana. The study area covers most of the Community Resource Management Areas - CREMAs (authorised communities to manage their natural resources for economic and livelihood benefits), and shares boundaries with the Mole National Park to the west. This CREMA includes Murugu, Kumbo Mognori, Kpulumbu, Bawena Wawato and Grubagu.

The annual rainfall is unevenly distributed and limited to six months (May-October). The mean annual rainfall ranges between $1000 \mathrm{~mm}$ and $1500 \mathrm{~mm}$ with its peak in July and September. Its average minimum temperature is about $24^{\circ} \mathrm{C}$ and average maximum temperature is about $40^{\circ} \mathrm{C}$. The dry season is characterized with the dry harmattan wind that starts in December and ends in March. This period is char- 


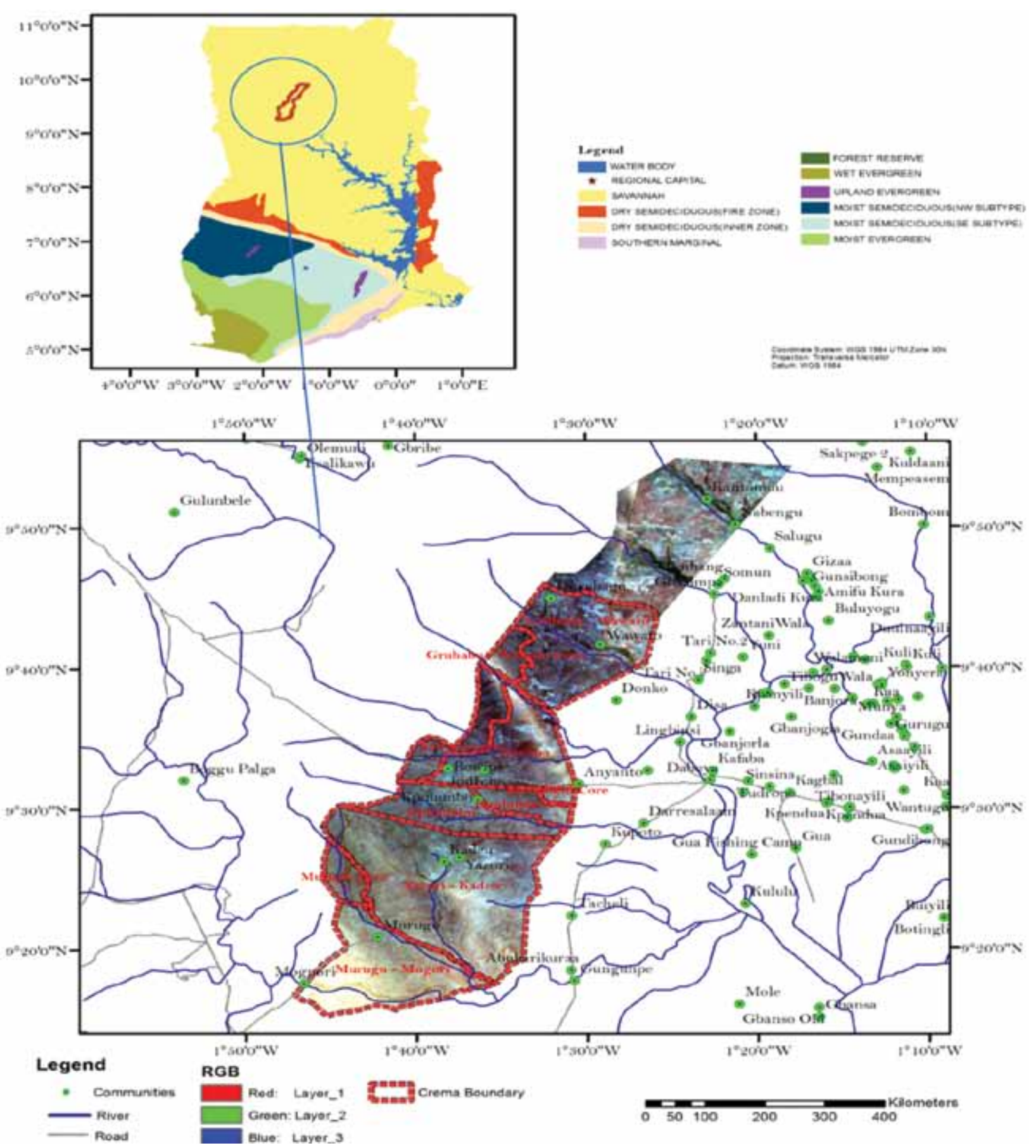

Figure 1. Map showing the descriptions of the study area

acterized by the annual bush burning either man-induced or natural phenomenon, which have hard devastating consequences on the vegetation, human lives and property.

\section{Materials and data}

Materials that were used in this study include:

- Satellite Imagery: The following satellite imageries were downloaded from the USGS portal based 
on their low cloud cover of less than $10 \%$ and the time frame of review. This includes: 2014 LandSat 8, 2010 ETM+, and TM 2000 and 1990 Images. To augment these imageries, 2014 NDVI products developed by the RMSC of FC were also acquired to be used in aiding the classification of the imageries. These images were initially geo-referenced in a UTM coordinate system.

- Aerial Photographs: 2010 Aerial photos covering the study area were obtained from the Survey and Mapping department of Land Commission of Ghana to act in the image classification of the 2010 epoch.

- Training data and 1990 and 2000 classified maps: The 2010 training data collected under the Forest Preservation Progamme (FPP) project and the 1990 and 2000 classified maps were obtained from the RMSC unit of the Forestry Commission of Ghana. These data were to be used to classify and validate the 2010, 2000 and 1990 satellite image.

- Topographic Map: A digital topographical map was acquired from the survey and the mapping department (SMD) of the Land Commission of Ghana (LC).

- GPS and Digital Camera: A Garmin hand-held GPS with camera were used during ground truth and verification data collections stages. The data acquired were helpful during the image classification and the accuracy assessment stages.

- Diameter tape, calliper, Vertex laser hypsometer: These instruments were useful in collecting the data during the forest inventory. Measurements such as diameter and height of trees were measured with diameter tape or callipers and laser hypsometer respectively.

- ERDAS Imagine, IDDRIS and ARCGIS Software: Selection of these software were based on of the aims and objectives of this project and the user friendliness of the software. Erdas imagine v10 was used in the classification of the imageries, while Idrisi Andes was used in predicting the future land-use change in the next 20 years based on scenarios and ArcGIS v10 was used in the modelling of the carbon content and the finalization of the map production.

\section{Methods}

The methods adopted in executing this study have been summarized in the flow chart (Fig. 2) and has been elaborated below.

\section{Image pre-processing}

Following the Standard Operating Procedure (SOP 003) for the acquisition of remote sensing data and generation of activity data (Forestry Commission of Ghana 2014), image rectification was the initial step undertaken to enhance the spectral resolution of various images. This method involves Image Enhancement technique (Normalize Equalizer), which was applied to the images to aid in better interpretation of the imageries. It should be noted that the $1990 \mathrm{TM}$ did have issues with cloud cover but was on the lower percentage $(>10 \%)$ as this was a criterion selected before downloading it from USGS and did not bring much issues to deal with.

Normalized Difference Vegetation Index (NDVI) was employed to classify the image into vegetated and non-vegetated (Forest and Non-forest) areas based on the values given in the ranges of -1 to $1,-1$ representing non-vegetated areas and towards 1 representing high vegetated areas. This helps in the classification of the imageries of the various epochs

It was realized from the source images that about 2-3 satellite images of each epoch (1990, 2000, 2010 and 2014) covers the study area. For this reason, subsetting was done to extract the area of interest (AOI) for each epoch.

\section{Image classification and Accuracy Assessment}

\section{Training data collections}

Before entering the field to collect data, a desktop review was carried out. All the relevant data and information concerning the study area were studied and analysed. The study area was carved out from the 2014 satellite image using the Area of Interest (AOI) and the topographical data was obtained from the digital map such as road network, river network and town dataset covering the study area, which were superimposed on the subsetted satellite image (Fig. 1). The resultant product was used to plan how the ground truth and verification data were to be collected. The study area was sub-divided into grids and numbered serially for easy sampling and identification of the sample areas. With this planned data and using the hand-held GPS coupled with digital camera, the locations of the proposed seven (7) various land-use categories and pictures of the corresponding locations were taken during the fieldwork to aid in the image classification process. 


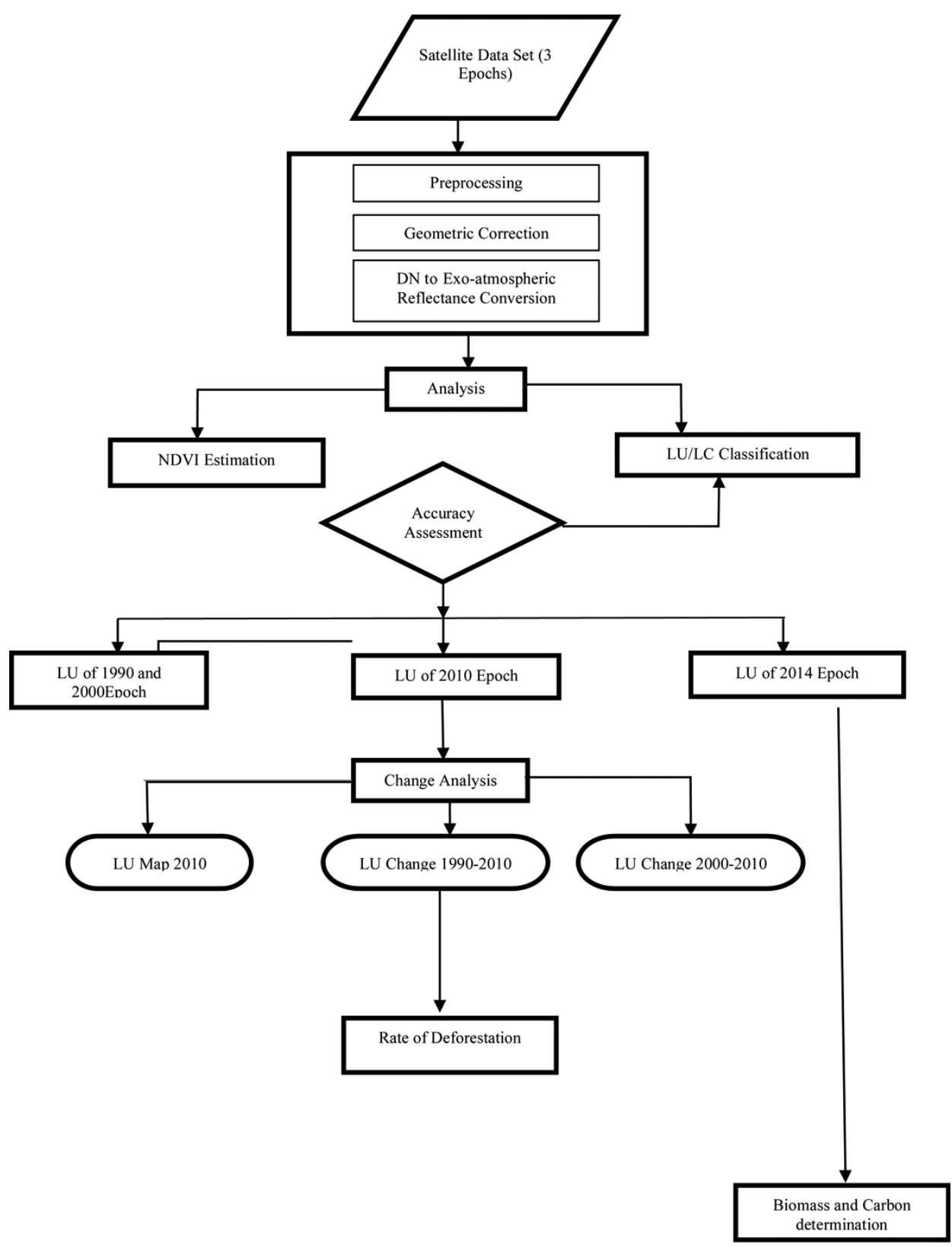

Figure 2. Flowchart indicating the procedure for the execution of the work

During the fieldwork, fifteen (15) to thirty (30) training classes per each land-cover/land-use type were collected depending on the variability in the locality within the study area. In all, a total of 220 training data were collected, 100 points were used as ground truth data for image classification and the remaining 120 data as verification data during the accuracy assessment.
Image Classification \& Accuracy Assessment

For the 2014 epoch, the field data were imported in Microsoft Excel and then divided into two (2) sets: 100 classes were used as training data and 120 as validation data. The training data combined with the generated NDVI image were used to run supervised classification based on the maximum likelihood classifier in Erdas imagine v10 software. The individual clas- 
Table 1. Land use description

\begin{tabular}{|l|l|}
\hline \multicolumn{1}{|c|}{ Landuse } & \multicolumn{1}{|c|}{ Description } \\
\hline Dense Woodland & $\begin{array}{l}\text { Natural woodland with canopy cover more than } 40 \% \text {, spatial coverage of more than one hectare } \\
\text { and tree height reaching } 5 \mathrm{~m} \text {. The stocking may vary from } 100 \text { to } 200 \mathrm{stems} / \text { ha. The trees have } \\
\text { short stems and profuse branching with DBH varying from } 10 \text { to } 70 \mathrm{~cm} \text {. They occur in large } \\
\text { stretches and show evidence of burning }\end{array}$ \\
\hline Open Woodland & $\begin{array}{l}\text { Natural woodland of canopy cover reaching 30\%, spatial area of one ha and tree height reaching } \\
5 \mathrm{~m} \text {. The tree diameter varies from } 10 \mathrm{~cm} \text { to } 40 \mathrm{~cm} \text { DBH. It is a tree grass mosaic and occurs in } \\
\text { disturbed natural woodlands, fallow areas and shows evidence of burning }\end{array}$ \\
\hline Riverine Forest & $\begin{array}{l}\text { Natural forest with closed canopy, mostly occurring in strips along rivers and streams and in some } \\
\text { wetlands }\end{array}$ \\
\hline Cropland & $\begin{array}{l}\text { Fallow areas and grass/crop mosaic mainly annual crops such as maize, cassava and yam amongst } \\
\text { others }\end{array}$ \\
\hline Grassland & $\begin{array}{l}\text { Long stretches of grass cover. Animal grazing occurs in these areas. The grasslands also occur in } \\
\text { wet and low-lying areas and sometimes along riverine belts }\end{array}$ \\
\hline Build-Up and Bare Surface & $\begin{array}{l}\text { These are areas that have been populated with permanent residence or covered with scanty grass, } \\
\text { exposed rocks and bare lands }\end{array}$ \\
\hline Water & Stagnant water, lakes, rivers and streams \\
\hline
\end{tabular}

Table 2. Error matrix

\begin{tabular}{|c|c|c|c|c|c|c|c|}
\hline \multicolumn{8}{|c|}{ Classification accuracy assessment report } \\
\hline Error matrix & \multicolumn{7}{|c|}{ Reference Data } \\
\hline Classified Data & $\begin{array}{c}\text { Dense } \\
\text { Woodland }\end{array}$ & $\begin{array}{c}\text { Open } \\
\text { Woodland }\end{array}$ & Riverine & Grassland & Cropland & $\begin{array}{c}\text { Built-Up/Bare } \\
\text { Surface }\end{array}$ & Water \\
\hline Dense Woodland & 20 & 2 & 3 & 0 & 0 & 0 & 0 \\
\hline Open Woodland & 3 & 50 & 4 & 3 & 0 & 0 & 0 \\
\hline Riverine & 2 & 0 & 8 & 0 & 0 & 0 & 0 \\
\hline Grassland & 0 & 2 & 0 & 6 & 0 & 0 & 0 \\
\hline Cropland & 0 & 0 & 0 & 0 & 10 & 0 & 0 \\
\hline Built-Up / Bare Surface & 0 & 0 & 0 & 0 & 0 & 7 & 0 \\
\hline Water & 0 & 0 & 0 & 0 & 0 & 0 & 0 \\
\hline Column Total & 25 & 54 & 15 & 9 & 10 & 7 & 0 \\
\hline \multicolumn{8}{|l|}{ Accuracy totals } \\
\hline Class & Reference & Classified & Number & Producers & Users & Kappa & \\
\hline Name & Totals & Totals & Correct & Accuracy & Accuracy & Statistics & \\
\hline Dense Woodland & 25 & 25 & 20 & $80.00 \%$ & $80.00 \%$ & 0.747 & \\
\hline Open Woodland & 54 & 60 & 50 & $92.59 \%$ & $83.33 \%$ & 0.697 & \\
\hline Riverine & 15 & 10 & 8 & $53.33 \%$ & $80.00 \%$ & 0.771 & \\
\hline Grassland & 9 & 8 & 6 & $66.67 \%$ & $75.00 \%$ & 0.730 & \\
\hline Cropland & 10 & 10 & 10 & $100.00 \%$ & $100.00 \%$ & 1.000 & \\
\hline Built-Up / Bare Surface & 7 & 7 & 7 & $100.00 \%$ & $100.00 \%$ & 1.000 & \\
\hline Water & 0 & 0 & 0 & - & - & 0.000 & \\
\hline Totals & 120 & 120 & 101 & & & & \\
\hline
\end{tabular}

Overall Classification Accuracy $=84.17 \%$; Overall Kappa Statistics $=0.7757$. 
sified scenes covering the study area were therefore mosaicked to obtain the 2014 classified image. In all, seven (7) land-use classes were generated within the study area in conformity with the Intergovernmental Panel on Climate Change (IPCC, 2003) for Land-use and Land-use Change and Forestry (LULUCF) definitions. Table 1 shows the various land-use classes with its descriptions.

Likewise, for the 2010 epoch, with the help of the training data coupled with the 2010 aerial photos obtained from RMSC under FPP project and fore-knowledge about the area, the 2010 imageries were classified based on maximum likelihood algorithm to obtain the seven (7) land-use classes within the study area. Here too the individual classified scenes were mosaicked to obtain the 2010 classified image. The validation data coupled with the aerial photos were used to verify the 2014 and 2010 classified images during the accuracy assessment process in Erdas imagine v10, based on the use of error matrix and kappa statistics (see Tab. 2 for the 2014 error matrix generated).

These classified images together with the two thematic maps obtained from RMSC were used to classify the 2000 and 1990 epochs. It should be noted that since there were no ground truth data, accuracy assessment could not be carried out on the 2000 and 1990 epochs. These resultant products were later opened in ArcMap v10 to draft and compose the various thematic maps (Fig. 3).

\section{Change Detection \& Analysis}

The land-use and land-use change analysis were performed to know the changes in terms of the spatial extent, trend and trajectory of the various land-use classes within the 20-year period (1990-2010). This was made possible by the use CrossTab module in Idrisi software. For the Idrisi software to accept these classified images - 1990, 2000, 2010 from Erdas imagine (.img file), the classified images were converted to raster file (.rst file) which is a recognized file in Idrisi. Three (3) change periods were considered under this study: 1990-2000, 2000-2010 and 1990-2010. The CrossTab module in Idrisi accept only two inputs, the former and the current epochs; so, for the change matrix 1990-2000, the 1990 and 2000 classified images were used to generate the 1990-2000 change matrix. The rest of the two change matrixes 2000-2010 and 1990-2010 were also generated for the two (2) time periods. These are shown in Tables 3, 4, 5 and 6, and Figures 4, 5 and 6.

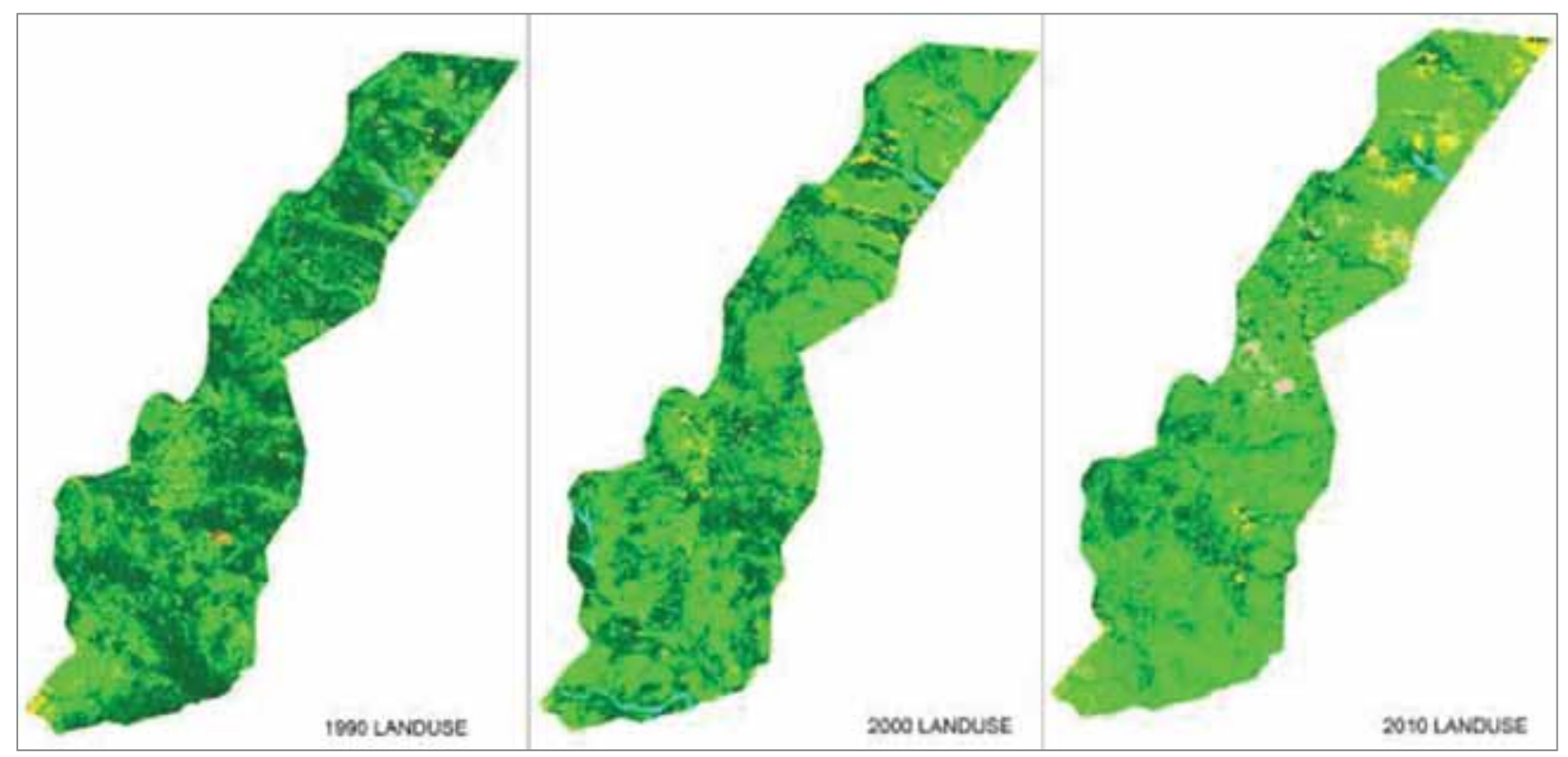

Figure 3A. Land-use map of the study area for the 3 epochs 


\section{DE GRUYTER \\ OPEN}

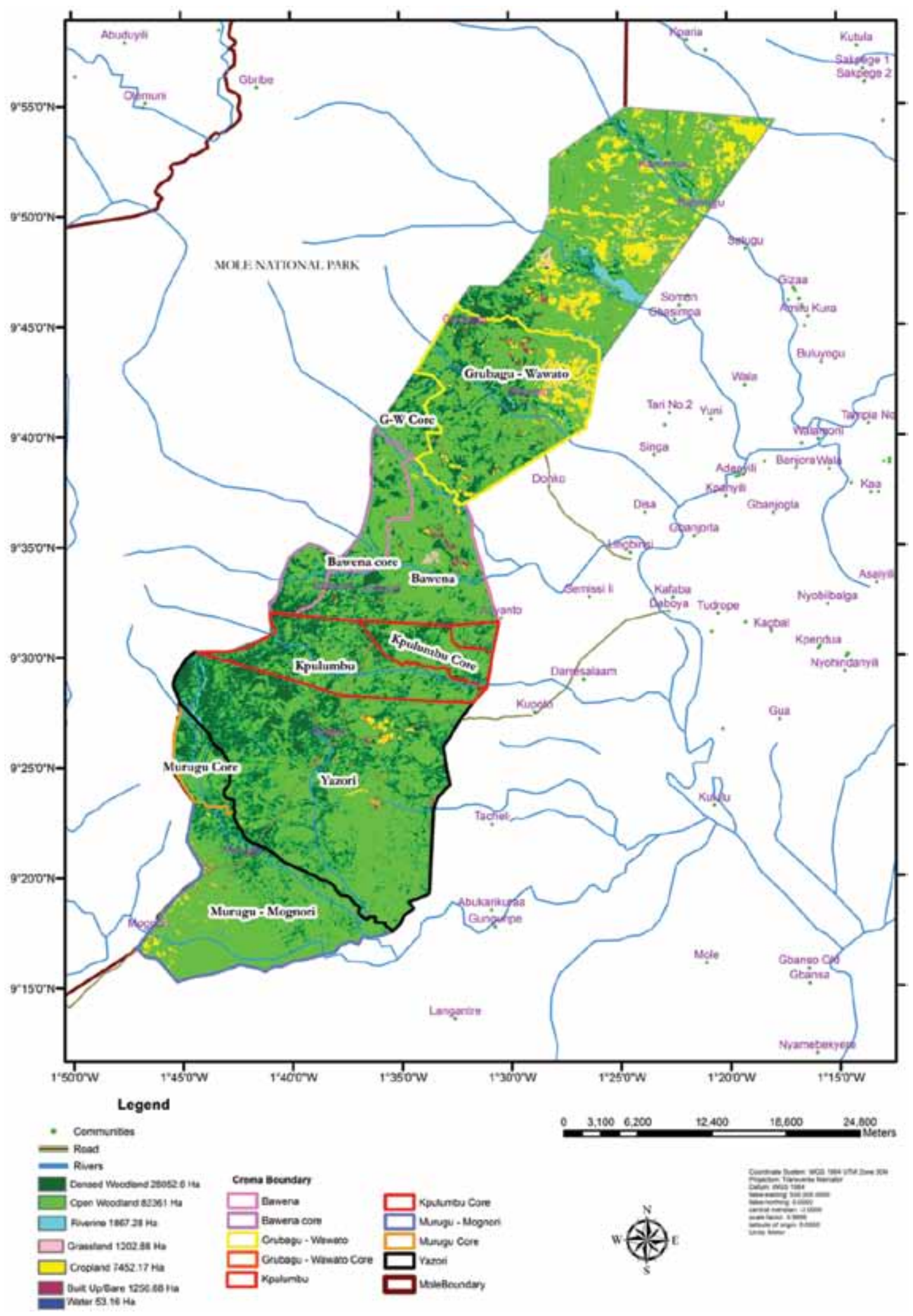

Figure 3B. 2014 Land use Map 
Table 3. Land-use change over a twenty-year period

\begin{tabular}{|c|c|c|c|c|c|c|}
\hline & \multicolumn{2}{|c|}{1990} & \multicolumn{2}{|c|}{2000} & \multicolumn{2}{|c|}{2010} \\
\hline & area (ha) & area $(\%)$ & area (ha) & area $(\%)$ & area (ha) & area $(\%)$ \\
\hline Dense WL & $68,998.30$ & $56.44 \%$ & $4,6041.1$ & $37.66 \%$ & $27,280.30$ & $22.32 \%$ \\
\hline Open WL & $43,712.65$ & $35.76 \%$ & $64,631.44$ & $52.87 \%$ & $77,657.80$ & $63.53 \%$ \\
\hline Riverine & $2,518.11$ & $2.06 \%$ & $3,690.27$ & $3.02 \%$ & $1,880.37$ & $1.54 \%$ \\
\hline Grassland & $3,412.44$ & $2.79 \%$ & $2,271.96$ & $1.86 \%$ & $6,755.76$ & $5.53 \%$ \\
\hline Cropland & $2,938.95$ & $2.40 \%$ & $4,801.23$ & $3.93 \%$ & $6,979.68$ & $5.71 \%$ \\
\hline Built-Up & 647.28 & $0.53 \%$ & 793.17 & $0.65 \%$ & $1,685.97$ & $1.38 \%$ \\
\hline Water & 12.24 & $0.01 \%$ & 10.80 & $0.01 \%$ & 0.09 & $0.00 \%$ \\
\hline TOTAL & $122,239.97$ & $100.00 \%$ & $122,239.97$ & $100.00 \%$ & $122,239.97$ & $100.00 \%$ \\
\hline
\end{tabular}

Table 4. Land-use matrix 1990-2000

\begin{tabular}{|c|c|c|c|c|c|c|c|c|c|c|c|}
\hline & \multicolumn{9}{|c|}{$1990 \mathrm{LU}$} & \multicolumn{2}{|c|}{ Gain } \\
\hline \multirow{9}{*}{$2000 \mathrm{LU}$} & $\mathrm{LU}$ & $\begin{array}{c}\text { Dense } \\
\text { WL }\end{array}$ & $\begin{array}{c}\text { Open } \\
\text { WL }\end{array}$ & Riverine & $\begin{array}{l}\text { Gras- } \\
\text { sland }\end{array}$ & $\begin{array}{l}\text { Cro- } \\
\text { pland }\end{array}$ & $\begin{array}{c}\text { Built- } \\
\text { Up }\end{array}$ & Water & Total & ha & $\%$ \\
\hline & Dense WL & $27,925.74$ & $15,349.86$ & 1213.92 & 586.44 & 963.18 & 0 & 1.98 & $46,041.12$ & $18,115.38$ & $39.35 \%$ \\
\hline & Open WL & $35,391.96$ & $25,221.51$ & 354.69 & 2413.44 & 1270.17 & 0 & 0.18 & $64,651.95$ & $39,430.44$ & $60.99 \%$ \\
\hline & Riverine & $2,336.49$ & 428.49 & 897.12 & 5.31 & 8.91 & 0 & 9.72 & $3,686.04$ & $2,788.92$ & $75.66 \%$ \\
\hline & Grassland & $1,284.93$ & 701.01 & 16.11 & 87.39 & 175.59 & 0 & 0 & $2,265.03$ & $2,177.64$ & $96.14 \%$ \\
\hline & Cropland & $1,970.37$ & $2,030.04$ & 30.69 & 270.90 & 489.87 & 0 & 0 & $4,791.87$ & $4,302,00$ & $89.78 \%$ \\
\hline & Built-Up & 15.12 & 62.37 & 0 & 44.01 & 24.39 & 647.28 & 0 & 793.17 & 145.89 & $18.39 \%$ \\
\hline & Water & 3.6 & 4.05 & 2.79 & 0.27 & 0 & 0 & 0.09 & 10.80 & 10.71 & $99.17 \%$ \\
\hline & Total & $68,928.21$ & $43,797.33$ & 2515.32 & 3407.76 & 2932.11 & 647.28 & 11.97 & $122,239.98$ & & \\
\hline \multirow{2}{*}{ Loss } & $\mathrm{Ha}$ & $41,002.47$ & $18,575.82$ & 1618.2 & 3320.37 & 2442.24 & 0 & 11.88 & $66,970.98$ & $54.79 \%$ & Change \\
\hline & $\%$ & $59.49 \%$ & $42.41 \%$ & $64.33 \%$ & $97.44 \%$ & $83.29 \%$ & $0.00 \%$ & $99.25 \%$ & & & \\
\hline Net change & $\%$ & $-20.14 \%$ & $18.58 \%$ & $11.33 \%$ & $-1.29 \%$ & $18.39 \%$ & $6.48 \%$ & $-0.08 \%$ & 55269 & $45.21 \%$ & Persist \\
\hline
\end{tabular}

Table 5. Land-use matrix 2000-2010

\begin{tabular}{|c|c|c|c|c|c|c|c|c|c|c|c|}
\hline & \multicolumn{9}{|c|}{$2000 \mathrm{LU}$} & \multicolumn{2}{|c|}{ Gain } \\
\hline \multirow{9}{*}{2010 LU } & LU & $\begin{array}{c}\text { Dense } \\
\text { WL }\end{array}$ & $\begin{array}{c}\text { Open } \\
\text { WoodL }\end{array}$ & Riverine & $\begin{array}{l}\text { Gras- } \\
\text { sland }\end{array}$ & $\begin{array}{l}\text { Cro- } \\
\text { pland }\end{array}$ & $\begin{array}{l}\text { Built- } \\
\text { Up }\end{array}$ & Water & Total & ha & $\%$ \\
\hline & Dense WL & $11,668.86$ & $13,086.63$ & 1290.60 & 252.63 & 977.85 & 0 & 3.78 & $27,280.35$ & 15.611 .49 & $57.23 \%$ \\
\hline & Open WL & $29,554.83$ & $42,265.17$ & 1249.65 & 1593.18 & 2989.98 & 0 & 4.95 & $77,657.76$ & $35,392.59$ & $45.58 \%$ \\
\hline & Riverine & 615.06 & 112.59 & 1125,00 & 1.80 & 23.49 & 0 & 0.81 & $1,878.75$ & 753.75 & $40.12 \%$ \\
\hline & Grassland & $2,304.09$ & $3,800.16$ & 16.65 & 257.49 & 372.69 & 0 & 1.26 & $6,752.34$ & $6,494.85$ & $96.19 \%$ \\
\hline & Cropland & $1,646.91$ & $4,839.03$ & 5.13 & 141.84 & 351.99 & 0 & 0 & $6,984.9$ & $6,632.91$ & $94.96 \%$ \\
\hline & Built-Up & 217.26 & 568.08 & 0 & 18.72 & 88.56 & 793.17 & 0 & $1,685.79$ & 892.62 & $52.95 \%$ \\
\hline & Water & 0 & 0 & 0.09 & 0 & 0 & 0 & 0 & 0.09 & 0.09 & $100.00 \%$ \\
\hline & Total & 46007.01 & $64,671.66$ & 3687.12 & 2265.66 & 4804.56 & 793.17 & 10.80 & $122,239.98$ & & \\
\hline \multirow{2}{*}{ Loss } & ha & $34,338.15$ & $22,406.49$ & 2562.12 & 2008.17 & 4452.57 & 0 & 10.8 & $65,778.3$ & $53.81 \%$ & Change \\
\hline & $\%$ & $74.64 \%$ & $34.65 \%$ & $69.49 \%$ & $88.64 \%$ & $92.67 \%$ & $0.00 \%$ & $100.00 \%$ & & & \\
\hline Net change & $\%$ & $-17.41 \%$ & $10.93 \%$ & $-29.37 \%$ & $7.55 \%$ & $5.2952 \%$ & $0.29 \%$ & $0.00 \%$ & $56,461.68$ & $46.19 \%$ & Persist \\
\hline
\end{tabular}




\section{DE GRUYTER}

\section{OPEN}

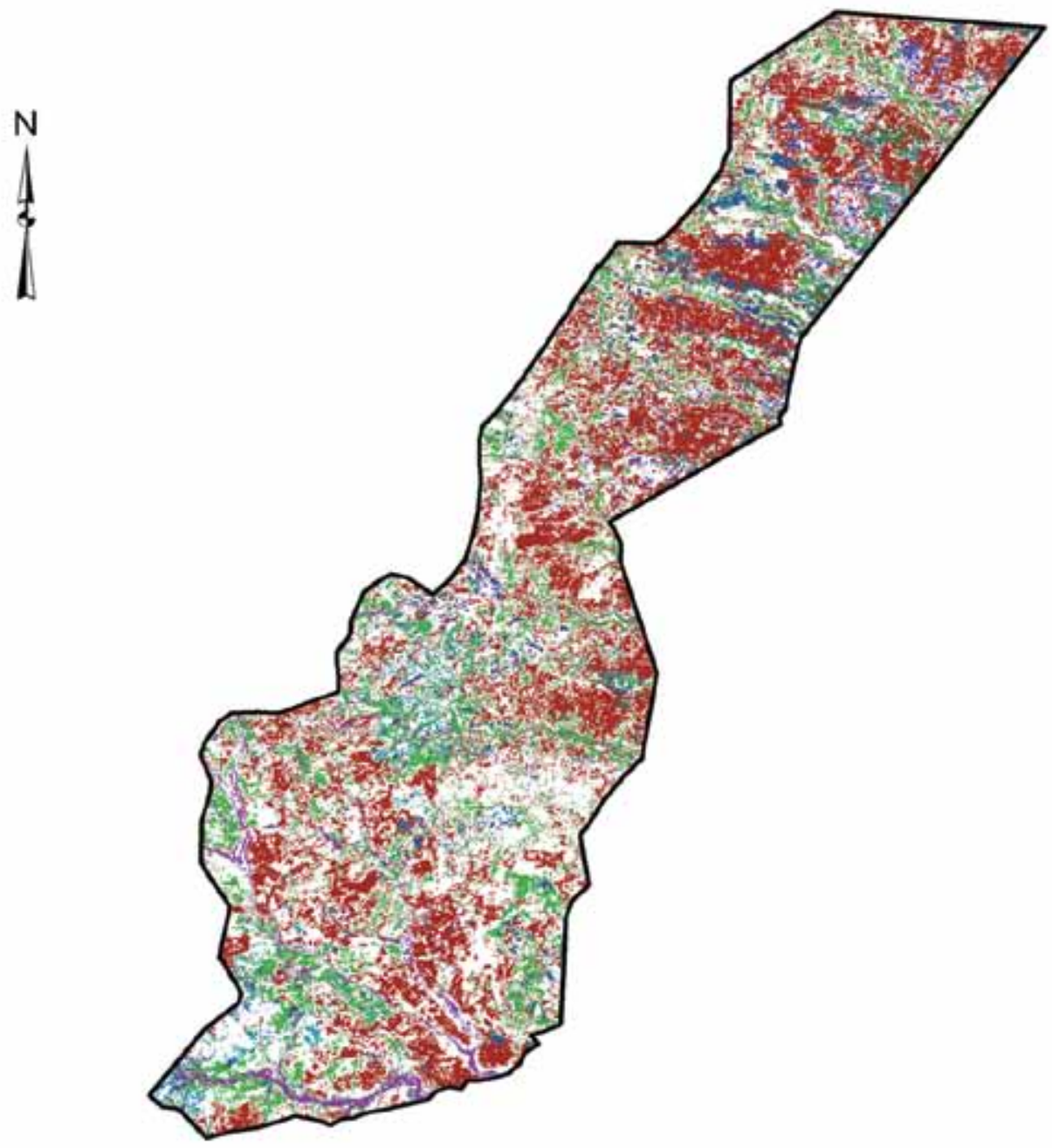

Legend
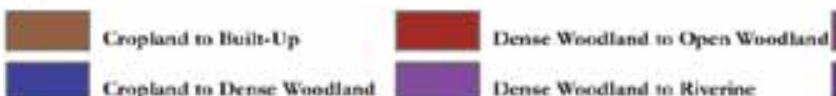

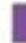

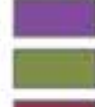

Bense Woodland is Kiverine

Copland to Ciraseland

Copland no Open Woedliand

Croplaad to Miverine

$\square$ Dense Woeslland to Built - L'P

Dense Weedland to Croplased

Dense Woedland to Girmalund

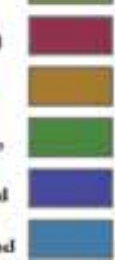

Denee Wendland to Water

Grasuland oo Ruils-Up

Gratuland to Cropland

Gravelund to Denee Woodland

Graulend as Open Woodland

Cirnaland to Riverine
Greasland to Watet

Open Wendland to Huilt-1/p

Open Wenelland to Croplanal

Open Woudtand ne Deme Winedland

Open Worelland to Graseland

Opes Woodland to Rinveine

Opes Weodlend to Wanter

Riverise to Cropland
Mizerine to Dease Woodland

Mirerine in Girassland

Riverine to Open Wendland

Mirerine to Water

Water te Denee Weodland

Water to Open Weodland

Whater so Rhereine

Nis Changr

Figure 4. Land-use conversion between 1990 and 2000 


\section{OPEN}

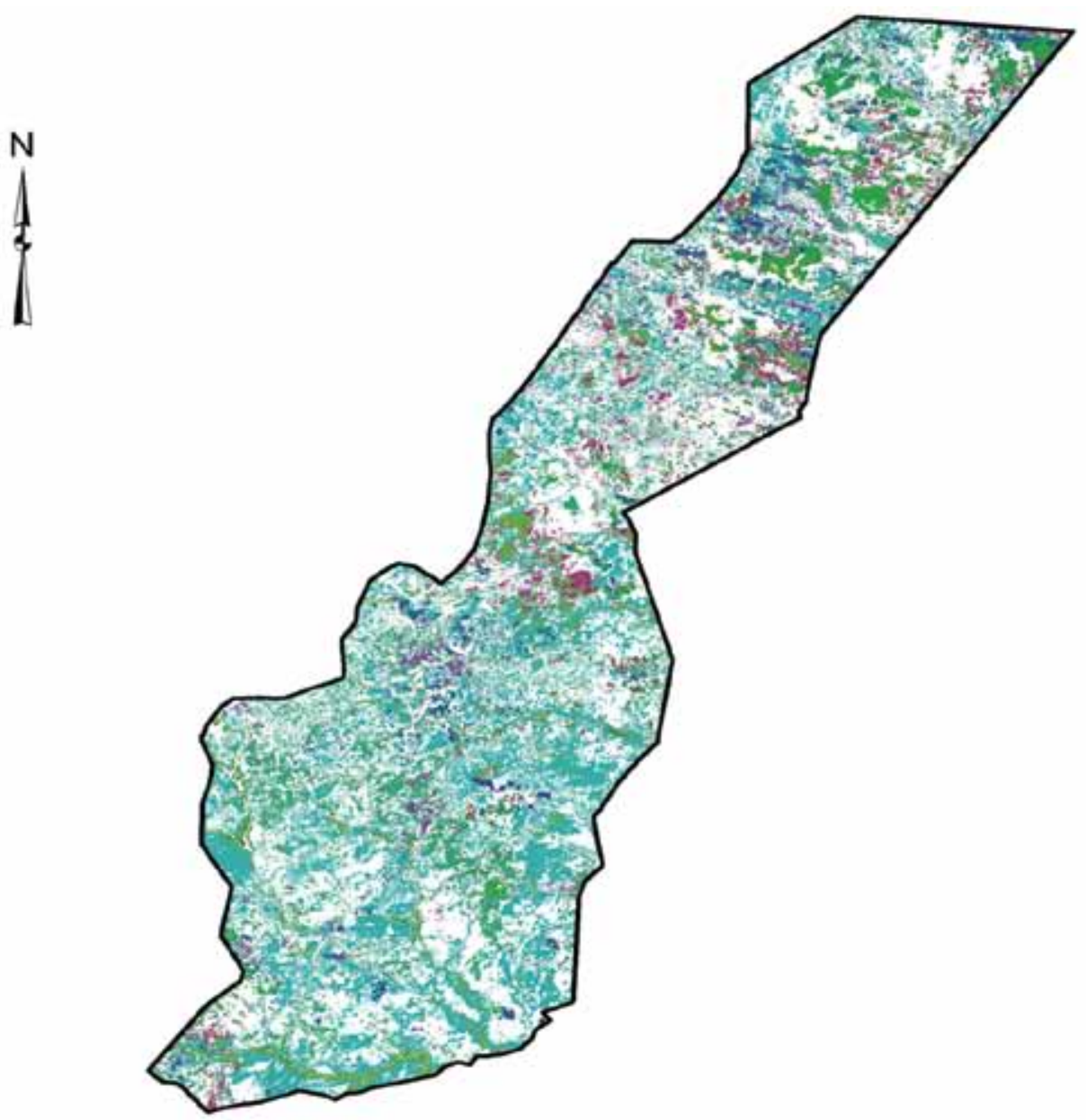

Lagenal
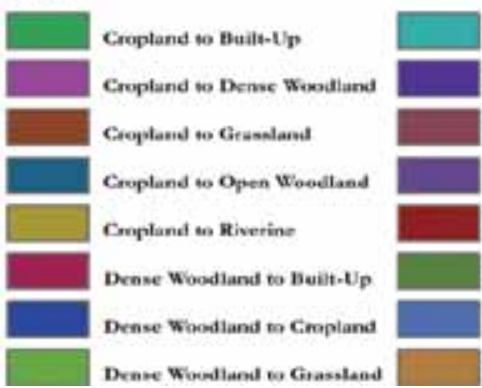

Dense Woodland to Open Woothand Dense Wrodland to Biveriac

Grasslaisd to Buih-t/

Grasetand te Cropland

Graseland ta Derner Wonellenat

Grasstand te Open Woodtand

Grastland te Misrine

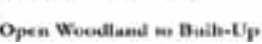

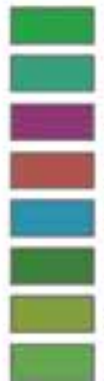

Open Woodland to Cropland

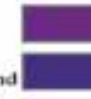

Riverine to Water

Water to Denee Woodlanil

Open Wontleand to Grassland

Open Woodland wo Rivetime

niwrine to Coppland

Miverine to Denee Woodland

Riverine bo Grassland

Mireriee 60 Open Wroulland

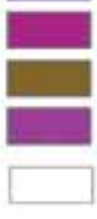

Water to Geasslaind

Water to Open Wuodland

Figure 5. Land-use conversion between 2000 and 2010 


\section{DE GRUYTER}

\section{OPEN}
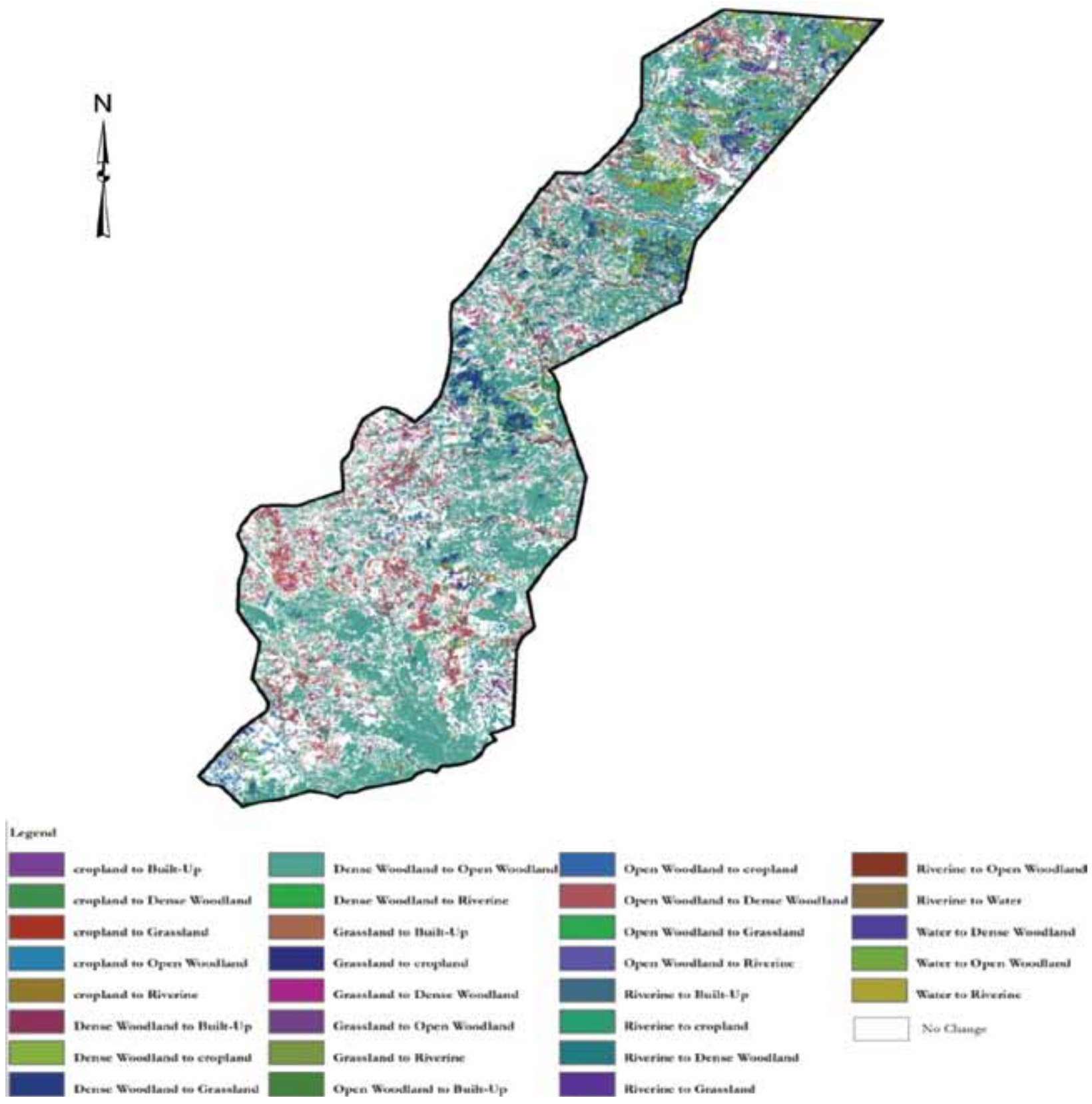

Figure 6. Land-use conversions for the period 1990-2010 
Table 6. Land-use matrix 1990-2010

\begin{tabular}{|c|c|c|c|c|c|c|c|c|c|c|c|}
\hline & \multicolumn{9}{|c|}{$1990 \mathrm{LU}$} & \multicolumn{2}{|c|}{ Gain } \\
\hline \multirow{9}{*}{$2010 \mathrm{LU}$} & LU & $\begin{array}{c}\text { Dense } \\
\text { WL }\end{array}$ & $\begin{array}{c}\text { Open } \\
\text { WL }\end{array}$ & Riverine & $\begin{array}{l}\text { Gras- } \\
\text { sland }\end{array}$ & $\begin{array}{l}\text { Cro- } \\
\text { pland }\end{array}$ & $\begin{array}{l}\text { Built- } \\
\text { Up }\end{array}$ & Water & Total & ha & $\%$ \\
\hline & Dense WL & $14,932.98$ & $10,499.04$ & 887.49 & 385.56 & 574.38 & 0 & 0.9 & $27,280.35$ & $12,347.37$ & $45.26 \%$ \\
\hline & Open WL & $44,755.20$ & $28,086.48$ & 707.31 & 2295.27 & 1802.52 & 0 & 10.98 & $77,657.76$ & $49,571.28$ & $63.83 \%$ \\
\hline & Riverine & 659.70 & 365.22 & 810.72 & 10.53 & 33.93 & 0 & 0.27 & $1,880.37$ & $1,069.65$ & $56.89 \%$ \\
\hline & Grassland & $4,147.02$ & $2,179.98$ & 57.15 & 233.82 & 137.79 & 0 & 0 & $6,755.76$ & $6,521.94$ & $96.54 \%$ \\
\hline & Cropland & $3,914.91$ & $2,289.24$ & 51.21 & 399.42 & 324.90 & 0 & 0 & $6,979.68$ & $6,654.78$ & $95.35 \%$ \\
\hline & Built-Up & 489.96 & 387.63 & 3.78 & 88.47 & 68.22 & 647.91 & 0 & $1,685.97$ & $1,038.06$ & $61.57 \%$ \\
\hline & Water & 0 & 0 & 0.09 & 0 & 0 & 0 & 0 & 0.09 & 0.09 & $100.00 \%$ \\
\hline & Total & $68,899.77$ & $43,807.59$ & $2,517.75$ & 3413.07 & 2941.74 & 647.91 & 12.15 & $122,239.98$ & & \\
\hline \multirow{2}{*}{ Loss } & ha & $53,966.79$ & 15721.11 & 1707.03 & 3179.25 & 2616.84 & 0 & 12.15 & 77203.17 & $63.16 \%$ & Change \\
\hline & $\%$ & $78.33 \%$ & $35.89 \%$ & $67.80 \%$ & $93.15 \%$ & $88.96 \%$ & $0.00 \%$ & $100.00 \%$ & & & \\
\hline Net Change & $\%$ & $-33.07 \%$ & $27.95 \%$ & $-10.91 \%$ & $3.39 \%$ & $6.39 \%$ & $6.157 \%$ & $0.00 \%$ & 45036.81 & $36.84 \%$ & Persist \\
\hline
\end{tabular}

\section{Determination of Rate of Deforestation}

In order to determine the rate of deforestation within the 20 years period (1990-2010), the three (3) classified images were recoded into two (2):

- Forested areas comprising of Dense Woodland, Open Woodland and Riverine Forest and

- Non-forest areas comprising of Grassland, Built$\mathrm{Up} /$ Bare ground and Water.

The rate of deforestation was also determined for the period $1990-2010$ by using the cross-tabulation method from the 1990 and 2010 recoded thematic maps. The result is shown in a transition matrix (Tab. 7).

Table 7. Rate of deforestation from 1990-2010

\begin{tabular}{|l|l|r|c|r|}
\hline \multicolumn{2}{|c|}{} & \multicolumn{2}{|c|}{1990 Epoch } & \\
\cline { 3 - 5 } \multicolumn{2}{|c|}{} & Forest & Non-Forest & 2010 Total \\
\hline \multirow{2}{*}{$\begin{array}{l}\text { Epoch } \\
\text { E10 }\end{array}$} & Forest & $101,704.14$ & $5,114.34$ & $106,818.48$ \\
\cline { 2 - 5 } & Non-Forest & $13,520.94$ & $1,900.53$ & $15,421.47$ \\
\hline & 1990 Total & $115,225.08$ & $7,014.87$ & $122,239.95$ \\
\hline \multicolumn{2}{|l|}{ Rate of Deforestation } & 0.111 & & \\
\hline
\end{tabular}

\section{Carbon Stock Assessment}

This study adopted the direct tree inventory measurement to determine the biomass content within each sample plot. The following procedures were adopted to determine the carbon stock within the study area.
Plot size and shape for carbon resource

\section{assessment}

Based on the 2014 land-use map, locations of sample plots were randomly selected and distributed proportionately according to the coverage and variability of the land-use classes within the study area in accordance to the Standard Operating Procedure (SOP 003) for the acquisition of remote sensing data and the generation of activity data (Forestry Commission of Ghana 2014). Based on the extracted coordinates of the sample plots and with the help of the handheld GPS, the individual sample plots were visited on field. A sample plot consisted of two (2) nested plots comprising of the main $30 \mathrm{~m} \times 30 \mathrm{~m}$ and a sub-plot of $15 \mathrm{~m} \times 15 \mathrm{~m}$ as seen in Figure 7. The plot size was chosen as a factor of the pixel size of the Landsat image in accordance with SOP 003 reported by the Forestry Commission of Ghana. For easy identification of the 2 nested plots on field, a blue rope was used to mark the main $30 \mathrm{~m} \times 30 \mathrm{~m}$ and a yellow rope for the $15 \mathrm{~m} \times 15 \mathrm{~m}$. This produced a net plot sample area of $900 \mathrm{~m}^{2}(0.09 \mathrm{ha})$ for the $30 \mathrm{~m} \times 30 \mathrm{~m}$ and 0.0225 ha for the $15 \mathrm{~m} \times 15 \mathrm{~m}$ as described in Tables 8 and 9. For a fair representation of the various land-use in the development of the carbon map, 56 sample plots proportionately distributed among the land use types and across the project area were used. 


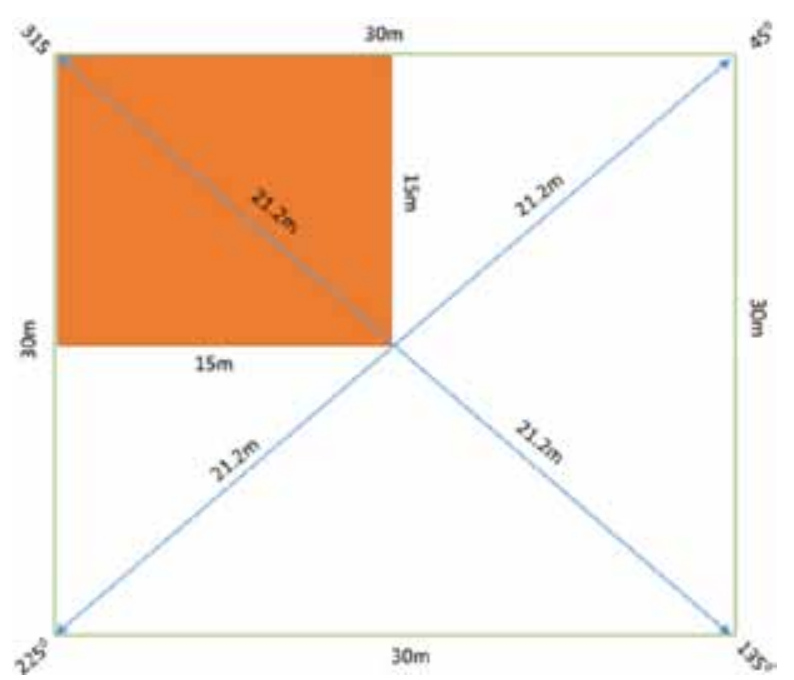

Figure 7. Nested Plot layout

Table 8. Carbon pool description

\begin{tabular}{|l|c|c|}
\hline \multicolumn{3}{|c|}{ Stand Level Area } \\
\hline Tree stand level & Stand dimension & Stand area (ha) \\
\hline Tally trees & $30 \mathrm{~m} \times 30 \mathrm{~m}$ & 0.0900 \\
\hline Dead standing & $30 \mathrm{~m} \times 30 \mathrm{~m}$ & 0.0900 \\
\hline Juvenile and other & $15 \mathrm{~m} \times 15 \mathrm{~m}$ & 0.0225 \\
\hline Down dead & $15 \mathrm{~m} \times 15 \mathrm{~m}$ & 0.0225 \\
\hline
\end{tabular}

Table 9. Tree diameter class

\begin{tabular}{|l|c|}
\hline & Diameter Class \\
\hline Tree category & Diameter $(\mathrm{cm})$ \\
\hline Tally trees & 10 and above \\
\hline Dead standing & 10 and above \\
\hline Juvenile and other & $2-9.99$ \\
\hline Down dead & 1 and above \\
\hline
\end{tabular}

\section{Tree inventory and measurement}

The savanna ecosystem is basically made of two layers of strata, namely the mature tall trees and juveniles trees of smaller diameter. So, in view of this, the $30 \mathrm{~m}$ $\times 30 \mathrm{~m}$ main plot size targeted all the standing trees and dead stems with the Diameter at Breast Height (dbh) 10 $\mathrm{cm}$ and above; and the other species with dbh ranges from $2 \mathrm{~cm}-9 \mathrm{~cm}$ were to be considered in the $15 \mathrm{~m} \times$ $15 \mathrm{~m}$ sub-plots.

Details of the below-mentioned data/parameters recorded during the fieldwork measurements as summarized below were converted to the plot-level estimates of aboveground biomass using models and scaled up to per hectare values. The tables show the above ground carbon pools considered (Tab. 10, 11 and 12).

Table 10. Plot data for biomass (tally trees)

\begin{tabular}{|c|c|c|c|c|c|c|c|c|}
\hline Id & Species Name & $\mathrm{DBH}(\mathrm{cm})$ & $\begin{array}{c}\text { Total } \\
\text { Height }(\mathrm{m})\end{array}$ & $\begin{array}{c}\text { Wood } \\
\text { Density }\end{array}$ & Comment & AGB $(\mathrm{kg})$ & $\begin{array}{l}\text { Total AGB } \\
\text { (tons) }\end{array}$ & $\begin{array}{c}\mathrm{AGC} \\
\text { (tons/ha) }\end{array}$ \\
\hline 1 & 2 & 3 & 4 & 5 & 6 & 7 & 8 & 9 \\
\hline 1 & Vitellaria paradoxa & 29.5 & 9.2 & 0.72 & & 667.08 & 9.74 & 108.23 \\
\hline 2 & Vitellaria paradoxa & 22.8 & 10.2 & 0.72 & & 387.36 & & \\
\hline 3 & Grewia molis & 49.5 & 11.3 & 0.65 & & 1794.76 & & \\
\hline 4 & Vitellaria paradoxa & 31.5 & 9.2 & 0.72 & & 766.09 & & \\
\hline 5 & Vitellaria paradoxa & 20.2 & 7.1 & 0.72 & & 300.04 & & \\
\hline 6 & Pterocarpus erinaceus & 11.4 & 6.2 & 0.94 & & 117.17 & & \\
\hline 7 & Pterocarpus erinaceus (A) & 13.1 & 6.9 & 0.94 & & 157.09 & & \\
\hline 8 & Pterocarpus erinaceus (B) & 15.3 & 7.1 & 0.94 & folked below & 217.97 & & \\
\hline 9 & Pterocarpus erinaceus & 12.0 & 6.9 & 0.94 & & 130.56 & & \\
\hline 10 & Pterocarpus erinaceus & 27.4 & 7.0 & 0.94 & & 745.26 & & \\
\hline 11 & Pterocarpus erinaceus & 11.7 & 7.1 & 0.94 & & 123.77 & & \\
\hline 12 & Pterocarpus erinaceus & 10.6 & 8.1 & 0.94 & & 100.49 & & \\
\hline 13 & Pterocarpus erinaceus & 11.4 & 7.2 & 0.94 & & 117.17 & & \\
\hline 14 & Vitellaria paradoxa & 19.2 & 7.1 & 0.94 & & 351.93 & & \\
\hline 15 & Vitellaria paradoxa & 24.7 & 7.9 & 0.94 & & 598.76 & & \\
\hline
\end{tabular}




\begin{tabular}{|c|l|c|c|c|c|c|c|c|}
\hline 1 & \multicolumn{1}{|c|}{2} & 3 & 4 & 5 & 6 & 7 & 8 & 9 \\
\hline 16 & Pterocarpus erinaceus & 10.7 & 9.0 & 0.94 & & 102.50 & & \\
\hline 17 & Pterocarpus erinaceus & 11.0 & 7.9 & 0.94 & & 108.66 & & \\
\hline 18 & Pterocarpus erinaceus & 11.7 & 8.0 & 0.94 & & 123.77 & & \\
\hline 19 & Pterocarpus erinaceus & 16.5 & 7.9 & 0.94 & & 255.62 & & \\
\hline 20 & Pterocarpus erinaceus & 14.5 & 8.1 & 0.94 & & 194.62 & & \\
\hline 21 & Pterocarpus erinaceus & 15.4 & 9.0 & 0.72 & & 169.27 & & \\
\hline 22 & Vitellaria paradoxa & 32.5 & 7.9 & 0.72 & & 818.31 & & \\
\hline 23 & Pterocarpus erinaceus (A) & 10.5 & 9.2 & 0.94 & Folked below & 98.50 & & \\
\hline 24 & Pterocarpus erinaceus & 16.8 & 9.5 & 0.94 & & 265.52 & & \\
\hline 25 & Vitellaria paradoxa & 20.6 & 7.9 & 0.72 & & 312.71 & & \\
\hline 26 & Vitellaria paradoxa & 30.5 & 8.5 & 0.72 & & 715.69 & & \\
\hline & & & & & & 9740.67 & & \\
\hline
\end{tabular}

Table 11. Plot data for biomass (juvenile trees)

\begin{tabular}{|c|c|c|c|c|c|c|c|c|}
\hline Id & Scientific name & $\begin{array}{l}\text { DBH } \\
(\mathrm{cm})\end{array}$ & $\begin{array}{l}\text { Height } \\
\text { (m) }\end{array}$ & W.D & $\begin{array}{c}\mathrm{AGB} \\
(\mathrm{kg})\end{array}$ & $\begin{array}{l}\text { Total AGB } \\
\text { (tons) }\end{array}$ & $\begin{array}{c}\text { AGB } \\
\text { (tons/ha) }\end{array}$ & $\begin{array}{c}\mathrm{AGC} \\
\text { (tons/ha) }\end{array}$ \\
\hline 1 & Burkea africana & 9.9 & 7.2 & 1.14 & 105.51 & 0.42 & 18.86 & 9.05 \\
\hline 2 & Bridelia micrantha & 2.5 & 1.2 & 0.64 & 3.25 & & & \\
\hline 3 & Vitellaria paradoxa & 4.2 & 1.8 & 0.72 & 10.92 & & & \\
\hline 4 & Burkea africana & 4.1 & 3.9 & 1.14 & 16.43 & & & \\
\hline 5 & Terminalia Avicinoidis & 2.5 & 1.5 & 0.90 & 4.57 & & & \\
\hline 6 & Terminalia Avicinoidis & 6.3 & 4.2 & 0.90 & 32.10 & & & \\
\hline 7 & Vitellaria paradoxa & 5.8 & 4.6 & 0.72 & 21.57 & & & \\
\hline 8 & Pileostigma venenosum & 3.8 & 3.2 & 0.79 & 9.70 & & & \\
\hline 9 & Terminalia Avicinoidis & 4.5 & 4.8 & 0.90 & 15.78 & & & \\
\hline 10 & Burkea africana & 3.4 & 1.9 & 1.14 & 11.07 & & & \\
\hline 11 & Terminalia Avicinoidis & 3.5 & 2.3 & 0.90 & 9.29 & & & \\
\hline 12 & Burkea africana & 3.7 & 2.8 & 1.14 & 13.23 & & & \\
\hline 13 & Bridelia micrantha & 5.4 & 3.7 & 0.64 & 16.49 & & & \\
\hline 14 & Bridelia micrantha & 5.5 & 4.2 & 0.64 & 17.14 & & & \\
\hline 15 & Lophira lanceolata & 6.0 & 3.1 & 0.90 & 28.96 & & & \\
\hline 16 & Bridelia micrantha & 4.0 & 1.7 & 0.64 & 8.75 & & & \\
\hline 17 & Pterocarpus erinaceus & 3.3 & 3.0 & 0.72 & 6.56 & & & \\
\hline 18 & Burkea africana & 7.7 & 6.2 & 1.14 & 62.09 & & & \\
\hline 19 & Burkea africana & 4.5 & 3.1 & 1.14 & 19.99 & & & \\
\hline 20 & Daniellia oliveri & 2.6 & 1.3 & 0.72 & 3.97 & & & \\
\hline 21 & Annona senegalensis & 2.5 & 1.5 & 0.40 & 2.03 & & & \\
\hline 22 & Annona senegalensis & 2.2 & 1.2 & 0.40 & 1.55 & & & \\
\hline 23 & Annona senegalensis & 2.6 & 1.3 & 0.40 & 2.20 & & & \\
\hline \multirow[t]{2}{*}{24} & Annona senegalensis & 2.0 & 0.8 & 0.40 & 1.27 & & & \\
\hline & & & & & 424.42 & & & \\
\hline
\end{tabular}


Table 12. Plot data for biomass (dead standing)

\begin{tabular}{|c|c|c|c|c|c|c|c|c|c|c|}
\hline Id & Species name & $\begin{array}{c}\text { DBH } \\
(\mathrm{cm})\end{array}$ & $\begin{array}{c}\text { Height } \\
(\mathrm{m})\end{array}$ & Comment & W.D & DC & AGB & $\begin{array}{c}\text { AGC } \\
(\mathrm{kg})\end{array}$ & $\begin{array}{c}\text { Total AGC } \\
(\text { tons })\end{array}$ & $\begin{array}{c}\text { AGC } \\
\text { (tons/ha) }\end{array}$ \\
\hline 1 & Terminalia Avicinoidis & 18.3 & 13.5 & no branches & 0.90 & 0.64 & 304.49 & 93.62 & 0.15 & 1.70 \\
\hline 2 & Pterocarpus erinaceus & 14.9 & 12.3 & small branches & 0.94 & 0.60 & 206.12 & 59.79 & & \\
\hline & & & & & & & & 153.42 & & \\
\hline
\end{tabular}

i. Tree ID: This involved the tallying system where one (1) was assigned to the first species identified at the beginning of the enumeration exercise and sequentially followed through to the end of the plot. Enumeration of trees started from the South West (SW) corner of the plot through the North West (NW) corner and then back down south and ends either in the North East (NE) or South East (SE) corner of the plot. The advantage of this approach is that it makes subsequent inventories easy, as one can always re-locate trees tallied in previous inventories even if the identification marks fall off with time.

ii. Local Name: The local and trade names of the identified species were recorded and for the species that could not be identified in the field - its information such as branches, leaves, bark slices and a photograph were taken to the herbarium of the Resource Management Support Centre of the Forestry Commission for analysis and identification.

iii. Tree diameter: All the trees with $\mathrm{DBH} \geq 10 \mathrm{~cm}$ (1.3 $\mathrm{m}$ above ground level) were measured and recorded using diameter tapes, whereas trees with DBH $<10$ were recorded using callipers. In order to avoid overestimation of the volume and to compensate for measurement errors, the diameter was measured in centimetres to one decimal precision. All juvenile trees with diameter between $5 \mathrm{~cm}$ and $10 \mathrm{~cm}$ were tallied and measured for both diameter and height for $15 \mathrm{~m} \times 15 \mathrm{~m}$ sub-plots. All the tree height measurements were measured with laser ace hypsometer or Vertex, and in circumstances where the tree was inclined, the base length was recorded with tape measure.

iv. Above ground Biomass and Carbon Stock Determination for Living Trees.

The above ground biomass (AGB) for tally trees, juvenile trees and seedlings were calculated using allo- metric equations developed in 2012 by Forest Research Institute of Ghana (FORIG) under the Forest Preservation Programme initiated by the Forestry Commission of Ghana (FC) in the PASCO 2013 report. The following allometric equations were used to calculate AGB per tree.

$$
\gamma=\rho \times 0.7342 \times\left(\mathrm{dbh}^{2}\right)^{1.0549}
$$

where:

$\gamma \quad$ - above ground biomass in $\mathrm{kg} /$ tree,

$\rho-$ wood density in $\mathrm{kg} / \mathrm{m}^{3}$,

$\mathrm{dbh}$ - diameter at breast height in $\mathrm{cm}$.

Equation 1 was used to calculate the aboveground biomass (AGB) for each tree using Excel spread sheet. The AGB per tree was then summed up for all the trees in the plot to obtain a stand-level AGB estimate. Each stand-level has a different area (ha), as shown in Table 1. The total stand-level AGB in kilograms of all the sample plots were converted to tonnes and further expressed in ton/ha by dividing the AGB by its stand area according to the formulae:

i. for matured trees ( $\mathrm{dbh} \geq 10 \mathrm{~cm}$ for $30 \mathrm{~m} \times 30 \mathrm{~m}$ plot) and

$$
\text { AGB }(\text { ton } / \text { ha })=\text { AGB } / 0.09
$$

ii. for juveniles $(2 \mathrm{~cm} \leq \mathrm{dbh}<10 \mathrm{~cm}$ for $15 \mathrm{~m} \times 15 \mathrm{~m}$ plot)

$$
\mathrm{AGB}(\mathrm{ton} / \mathrm{ha})=\mathrm{AGB} / 0.0225
$$

To obtain the above bround carbon (AGC), a standard carbon fraction value of 0.48 was applied to the AGB values for stand-level. This is expressed in equation 3 as:

$$
\mathrm{AGC}(\mathrm{ton} / \mathrm{ha})=0.48 \times \mathrm{AGB}
$$

Total AGC per plot was obtained by adding AGC (ton/ha) for tally trees, juvenile trees, seedlings and others. 
i. Determination of Carbon Stock for Dead Standing Trees

Standing dead trees are classified into 4 different classes based on the trees' decomposition level. The different levels are:

- tree with branches and twigs and resembles a live tree (except for leaves),

- tree with no twig, but with persistent small and large branches,

- tree with large branches only,

- bole (trunk) only, no branches.

The model for calculating the carbon of standing dead tree, as developed by FORIG, is expressed as:

$$
\mathrm{Z}(\mathrm{kg} / \text { tree })=0.48 \times \mathrm{DC} \times \mathrm{AGB}
$$

where:

$\mathrm{Z} \quad$ - above ground carbon in $\mathrm{kg}$ for dead standing tree,

AGB - above ground biomass from equation 1,

0.48 - carbon fraction,

DC - decomposition coefficient.

The DC is calculated as $\mathrm{DC}=\mathrm{D}^{2} \div[(2.95999+$ $+1.08769 \times \mathrm{D})]^{2}$ according to the Näslund's equations were used to calculate the decomposition coefficients for level (iii) and (iv). The AGC per dead standing tree was then summed up for all the dead standing trees to obtain the total AGC estimate. The total AGC (kg) was converted to tons and further expressed as ton/ha by dividing the tons by its stand area ( $0.09 \mathrm{ha})$.

Table 13. Plot data for biomass (down dead) ii. Determination of Carbon Stock for Downed Deadwood

Dead branches, stems, boles of trees and branches that have fallen and lie on or above the ground were considered for sub-plots of $15 \mathrm{~m} \times 15 \mathrm{~m}$ on every plot. Only the dead wood stems and their fragments with at least $2 \mathrm{~cm}$ thick lying within the plot extent were measured and recorded. The parameters measured were the length $(\mathrm{m})$ and diameters $(\mathrm{cm})$ at the two ends of the wood. The following observations were considered:

- Sound (blade does not sink or is bounced off) and has DL value of 1.0.

- Medium (blade partly sinks into the piece of wood or there has been some wood loss) and has DL value of 0.71 .

- Rotten (blade sinks well into the piece, there is extensive wood loss and the piece is crumbly) and has DL value of 0.31 .

The volume of downed deadwood (particle) is calculated using the frusto-conical formula:

$$
\text { Volume }=\pi \times \operatorname{LPX}(\mathrm{r} 21+\mathrm{r} 22+\mathrm{r} 1 \mathrm{r} 2) / 3
$$

Using wood density, decomposition level and carbon fraction, the volume is transformed into carbon as:

$$
\begin{gathered}
\mathrm{Z}(\mathrm{gr} / \text { particle })=\mathrm{DL} \times \mathrm{CF} \times \mathrm{WD} \times \\
\times \pi \times \mathrm{LP} \times 100 \times(\mathrm{r} 21+\mathrm{r} 22+\mathrm{r} 1 \mathrm{r} 2) / 3
\end{gathered}
$$

where:

Z - carbon per particle (downed deadwood) in grams, DL - decomposition level,

CF - carbon fraction $=0.48$,

\begin{tabular}{|c|c|c|l|l|l|l|l|c|c|c|c|c|c|c|c|c|}
\hline $\begin{array}{c}\text { Base } \\
\text { Dia } \\
(\mathrm{cm})\end{array}$ & $\begin{array}{c}\text { Tip } \\
\text { Dia } \\
(\mathrm{cm})\end{array}$ & $\begin{array}{c}\mathrm{L} \\
(\mathrm{m})\end{array}$ & $\begin{array}{c}\text { Com- } \\
\text { ment }\end{array}$ & W.D & D.L & $\begin{array}{c}\mathrm{C} \\
\text { frac- } \\
\text { tion }\end{array}$ & Pie & $\begin{array}{c}\text { Radius } \\
\text { at base } \\
\left(\mathrm{r}_{1}\right)\end{array}$ & $\begin{array}{c}\text { Radius } \\
\text { at tip } \\
\left(\mathrm{r}_{2}\right)\end{array}$ & $\mathrm{r}_{1} \wedge$ & $\mathrm{r}_{2} \wedge 2$ & $\mathrm{r}_{1} \mathrm{r}_{2}$ & $\begin{array}{c}\text { Sum } \\
\text { of rs/3 }\end{array}$ & C (gr) & $\begin{array}{c}\mathrm{C} \\
(\text { ton })\end{array}$ & $\begin{array}{c}\mathrm{C} \\
(\text { ton/ha) }\end{array}$ \\
\hline 4.5 & 4 & 1.29 & sound & 0.90 & 1.00 & 0.48 & 3.142 & 2.25 & 2.00 & 5.06 & 4.00 & 4.50 & 4.52 & 791.59 & 0.003 & 0.146 \\
\hline 3.8 & 3.3 & 1.14 & sound & 0.90 & 1.00 & 0.48 & 3.142 & 1.90 & 1.65 & 3.61 & 2.72 & 3.14 & 3.16 & 488.32 & & \\
\hline 3.6 & 3 & 0.65 & sound & 0.90 & 1.00 & 0.48 & 3.142 & 1.80 & 1.50 & 3.24 & 2.25 & 2.7 & 2.73 & 240.86 & & \\
\hline 3.5 & 2.7 & 0.92 & sound & 0.94 & 1.00 & 0.48 & 3.142 & 1.75 & 1.35 & 3.06 & 1.82 & 2.36 & 2.42 & 315.09 & & \\
\hline 5.5 & 4.2 & 0.81 & medium & 0.94 & 0.94 & 0.48 & 3.142 & 2.75 & 2.10 & 7.56 & 4.41 & 5.78 & 5.92 & 638.56 & & \\
\hline 3.1 & 1.6 & 1.10 & medium & 0.94 & 0.94 & 0.48 & 3.142 & 1.55 & 0.80 & 2.40 & 0.64 & 1.24 & 1.43 & 209.25 & & \\
\hline 4.3 & 1.7 & 1.31 & sound & 0.90 & 0.90 & 0.48 & 3.142 & 2.15 & 0.85 & 4.62 & 0.72 & 1.83 & 2.39 & 382.61 & & \\
\hline 2.7 & 1.5 & 1.41 & sound & 0.94 & 0.94 & 0.48 & 3.142 & 1.35 & 0.75 & 1.82 & 0.56 & 1.01 & 1.13 & 212.79 & & \\
\hline & & & & & & & & & & & & & & 3279.08 & & \\
\hline
\end{tabular}


WD - wood density,

LP - length of particle (m),

rl - radius at the base $(\mathrm{cm})$,

r2 - radius at the tip $(\mathrm{cm})$.

Equation 5 was used to calculate the carbon for each particle and subsequently summed up for all particles for the plot. The total carbon (gr) was converted to tons per hectare values.

The total carbon for dead standing trees was added to the total carbon for dead down trees to give the total dead carbon for the plot.

Grand total carbon per plot (ton/ha) was obtained by summing up the total carbon (ton/ha) for AGC (tally trees, juvenile trees, seedlings and others) and deadwood carbon (dead standing trees and downed deadwood). Tables 10-13 details the carbon stock calculated from the equations elaborated above.

iii. Carbon Map Production

Using ordinary kriging technique as a geo-statistical tool in ArcGIS, the carbon stock maps was generated for the study area. This technique was used because it provided more accurate and visually appealing map output. The database with plot number, the coordinates of each plot and the carbon values of each plot were recorded in MS excel sheet. This was imported into ArcGIS 10.0 and later applied a geo-statistics tool - kriging - to generate the carbon map for the study area.

\section{Results}

\section{Image Classification \& Accuracy Assessment}

From the classification and analysis of 2014 Landsat image (Fig. 3), seven main land use categories were identified: Dense Woodland, Open Woodland, Riverine Forest, Grassland, Cropland, Settlement/Bare Surface and Water. The study revealed that the Woodlands in the study area are made up of complex mosaic of dense woodland, open woodland tree-grass mosaic and gallery forest. These categories form the main carbon pools in the area and are qualified as forest under the national definition of forest as well as the recommendations of the draft Measuring, Reporting and Verification (MRV), as reported by the Forestry Commission of Ghana (Indufor Oy 2015). The FPP report (2012) was used as the basis in defining and categorizing the Woodlands.

\section{Accuracy assessment of Image Classification}

The accuracy of the classification was assessed using error matrix and Kappa statistic (Tab. 2) recommended by Lillesand and Kiefer (2008). This procedure is done to verify how accurate the classified images relate on the ground reference. In this project, a total of 120 ground verified points were used to perform the accuracy assessment of the 2014 classified map. The report is presented in an Error Matrix and Kappa statistics, which explains the agreement of land use classes to each other. Overall, an accuracy of $84.2 \%$ was obtained with a kappa statistic of 0.78 . The other epochs 1990 and 2000 could not be checked as there were no verified data available to carry out this method. From the accuracy assessment table, it can be deduced that the image classification accuracy meets the standard thresholds of $75 \%$ or above (Lillesand and Kiefer 2008) as well as SOP 003 recommended $80 \%$.

\section{Change Detection and Analysis}

The change analysis was performed on the 1990, 2000 and 2010 land use maps. This was done in conformity with the IPCC Good Practice Guidance (IPCC 2003), which lays emphasis on the use of time consistent data sets to determine and establish the baseline information and building historical data.

The open and dense woodlands represent the bulk of the biomass and carbon pools in the project area. The dense woodland in 1990 had the largest spatial extent of $68,998.30$ ha, representing $56 \%$ of the land area. However, this diminished to $46,041.10$ ha representing $37 \%$ in 2000 and again reduced to $27,280.30$ ha representing $22.32 \%$ in 2010 . On an average, whereas the dense woodland was losing cover at 2,295.72ha per annum, at the rate of 0.18 within $1990-2000$, it lost $18,760.80$ ha within 2000-2010, constituting a rate of 0.015 per annum. For the 20-year period, the dense woodland lost 2,085.9 ha, representing a rate of 0.017 per annum. The open woodland in 1990 had a spatial coverage of $43,712.65$ ha; however, these increased tremendously to $77,657.8$ ha at the rate of 0.27 per annum. This means that the dense woodlands were being degraded to open woodland, signifying the increasing phenomenon of forest degradation (Fig. 8). Riverine forest lost from 2,518 ha in 1990 to $1,880.37$ ha in 2010 (a difference of 125.91 ha) at an annual rate of 0.02 . However, considering the change over its size in 1990, the rate is 8.1 per annum, which is the highest rate of loss in the study area, signifying serious 
deforestation of the riverine forests. Dense woodland diminished at the rate of 0.5 over its area in 1990 and open woodland expanded at a rate of 0.357 . The land use trend over the 20 years at a 10 -year interval shows diminishing dense woodland and expansion in open woodland as well as grassland, a clear indication of loss of forest cover leading to deforestation. The area also witnessed a drastic loss in riverine forest and gradual expansion of settlements, which translated into expansion of croplands. Based on the national definition of forest, the deforestation rate for 20 years (1990-2010) for the study area is 0.11 , this is far lower than the average national deforestation rate of $2 \%$ estimated under the FPP project. This is elaborated in Table 3.

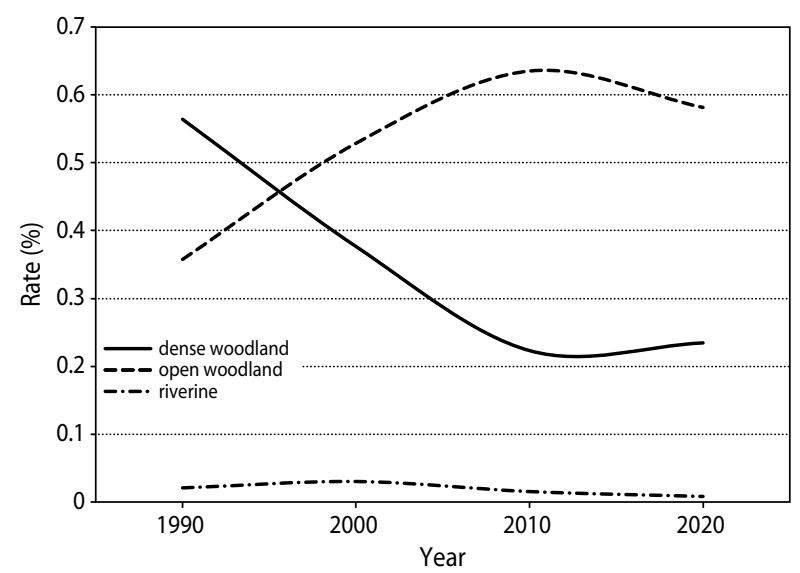

Figure 8. Rate of deforestation of woodland under Business As Usual scenario

The land use conversion (Fig. 4) and land use conversion matrix (Tab. 4) show the transfer of land use and the transition of the land use categories from one to the other. The diagonal values highlighted in grey show areas that did not change over the period. The values shown above the diagonal values in grey are the areas that have gained from the land use transfers, while the values below the diagonal show areas that have lost from the transfers. Over the 10 years period, $54.7 \%$ of the total areas experienced land use conversion, while $45 \%$ of the areas persisting in other words did not change between 1990 and 2000. Dense woodland suffered the highest loss of $20 \%$, followed by riverine, which recorded $11.33 \%$ loss within the 10 year interval. By implication, it means that the closed forest and riverine forest are being lost annually at $2 \%$ and $1.1 \%$ respectively. For the areas that gained, open forest recorded the highest gain of $18.58 \%$ followed by built-up of $18.39 \%$, while cropland expanded by $6.4 \%$ and grassland lost by $1.2 \%$. Generally, the fluxes in the land sector over the period were very high, making the area net emitter of GHG and good case for REDD+ intervention.

The land use conversion from 2000-2010 depicted in Figure 5 and Table 5 show an overall transfer of $53.87 \%$, while $46.19 \%$ of the land remained unchanged as highlighted in the green colour. Over the period, riverine recorded the highest net loss of $29.37 \%$ followed by dense woodland with a net loss of $17.41 \%$. Built up/ bare surface expanded by $52.97 \%$, almost double of its size from 1990 to 2000. This shows serious migration as a result of search for new farm lands or charcoal production. Grassland and cropland increased marginally by $7.5 \%$ and $2.3 \%$ respectively.

The land use conversion map (Fig. 6) and conversion matrix (Tab. 6) show that the fluxes in the area was $65 \%$, while $36.84 \%$ of the land area remained unchanged as highlighted in the green colour; the dense woodland recorded the highest net loss $78.33 \%$, while open forest recorded the highest net gain of $27 \%$. These were followed by riverine that recorded a net loss of $10.91 \%$. This was followed by cropland and built-up/ bare surfaces. Grassland gained marginally by $3.3 \%$ and water remained constant.

\section{Carbon Assessment}

Carbon stocks were estimated for three pools; aboveground carbon (AGC), and deadwood carbon (DWC) and the details are shown in Table 10, 11, 12 and 13; the total carbon stock within the study area is summarized in Table 14. Figure 9 shows the map of the carbon within the study area with grassland land-use class having the lowest carbon per hectare of $7 \mathrm{t} /$ ha measured and recorded to the highest carbon per hectare of $192.93 \mathrm{t} / \mathrm{ha}$ recorded for dense woodland. The study revealed that total carbon per township varies as different land-use categories show variation in the Above Ground Carbon (AGC) within the study area. For instance, the total AGC within Yezori township was recorded to be $108 \mathrm{t} / \mathrm{ha}$ being the lowest, followed by Wurubu township, which recorded as $115.468 \mathrm{t} / \mathrm{ha}$ and that of Gbarang township recorded as $133.69 \mathrm{t} / \mathrm{ha}$. Lastly, the highest AGC of $192.93 \mathrm{t} / \mathrm{ha}$ was recorded at Kpulumbu township (see Tab. 14 and Fig. 9 for details on the measured AGC). 
Table 14. Summary of carbon content in the study area

\begin{tabular}{|l|c|}
\hline \multicolumn{1}{|c|}{ Content (tree/plot) } & C (tons/ha) \\
\hline \multicolumn{2}{|c|}{ Aboveground Carbon } \\
\hline Tally & 73.14 \\
\hline Juvenile & 9.05 \\
\hline Sub-total (a) & 82.19 \\
\hline \multicolumn{2}{|c|}{ Deadwood Carbon } \\
\hline Dead standing & 1.70 \\
\hline Downed deadwood & 0.15 \\
\hline Sub-total (b) & 1.85 \\
\hline Grand Total of Carbon $(\mathrm{a}+\mathrm{b})$ & 84.04 \\
\hline
\end{tabular}

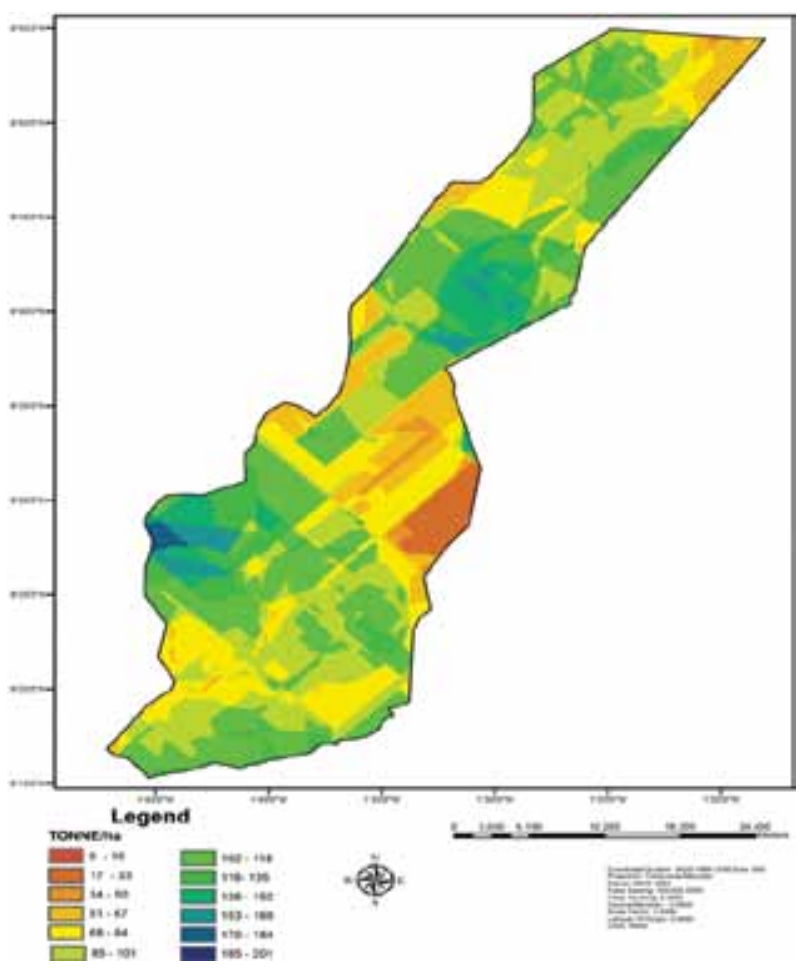

Figure 9. Carbon Map of the Study Area

\section{Discussion}

\section{Land-Use Categories}

\section{Dense woodland}

This land use class follows the open woodland with an annual deforestation rate of 0.18 and 0.015 referencing from 1990-2000 and 2000-2010 respectively. This gives an average annual deforestation rate of 0.017 for the twenty (20) year period, which is lower than the annual deforestation rate of closed forest estimated as 0.12 per annum reported by FPP (2012) and 0.3 per annum by the World Bank (2007) for Ghana. This suggests that the woodlands in the study area is much more stable than the high forest zone. This is further elaborated by the high average stems per hectare ranging from 200-400 stems, which gives approximate stems of 24,000-28,000 for an average compartment of 128 ha. This stocking level is very high compared to the current stocking level of good forest in the Western Region, which is around 10,000 stems per compartment (FPP 2012). This is indicative of the savannah woodlands having a high stocking level compared to the high forest zone but with smaller diameters. However, it is worth noting that the abundance of most hard woods in the High Forest Zone is threatened due to unsustainable exploitation over a long period according to the Indufor Oy Report 2015.

The depletion of the woodland is attributable to the harvesting of wood for commercial timber export, housing construction in expanding settlements, charcoal burning, agriculture and wildfires. The bulk of energy supply in Ghana is met from fuelwood that account for over $70 \%$ of the total primary energy supply (Indufor Oy Report 2015). The bulk of fuelwood amounting to $90 \%$ is obtained directly from the natural forest. The transitional zone and the Damongo district in particular, have been identified as providing the bulk of dense wood resources for fuelwood and timber export (Indufor Oy Report 2015). Consequently, the dense wood resources are depleting at a faster rate as a result of the unsustainable practices in the production and marketing of the products that incurs high levels of waste. If this trend of consumption continues, Ghana is likely to consume more than 25 million tonnes of fuel wood by the year 2020, which will lead to extinction/conversion of the woodlands to grassland. As a result of these activities, the dense woodland now exists in patches within the study area. According to the FPP (2012), the dense woodland holds an estimated $30-80 \%$ of carbon stock in the area and has diverse plant and animal species which contributes to more than $70 \%$ of the biodiversity resources of the study area.

Deforestation, as a result of indiscriminate burning and illegal timber harvesting in recent times, has contributed significantly to the destruction and fragmen- 
tation of the dense woodland, which has led to loss of habitat and breeding grounds, and isolation of the native and indigenous species. Wildfires remain one of the most important threats to the woodland (Mackay 2014; Srivastav and Srivastav 2015).

This study revealed that dense woodland has a diverse structure and form, as in some areas, the dense woodland are made of large diameter trees ranging from $60-120 \mathrm{~cm}$ with average tree stocking of 100-200 trees/ha and have a two-layer structure. The understory is a composition of grass and saplings, and occurs away from human settlements and farms. In some areas, the dense woodland is made of clustered trees of $30-80 \mathrm{~cm}$ with average stocking of 200-400 stems/ha, which are also far away from human settlements and farms; however, charcoal production and fuelwood harvesting is intensive in these areas. Fuelwood harvesting is done in such a way that farmers in very subtle manner use fire to kill trees by setting fire to the base of the trees, and by the next farming season, the trees die off and becomes firewood for harvesting. Also, the herdsmen set fires to promote the sprouting of fresh grass for grazing, thus contributing to conversion from dense woodland to open grassland. This has often generated serious land use conflict resulting in communal violence between the cattle herders who are mainly Fulani herdsmen and the indigenous crop farmers. This practice is the main driver of the conversion of the woodlands to grassland (FPP-PASCO-FC Report, 2013).

\section{Open woodland}

The open woodland is the transformation of the dense woodland as a result of charcoal production, farming activities, extensive and intensive timber harvesting, over grazing and annual bush fires. It forms a transition between the dense woodlands, agriculture and grassland, and therefore makes it a critical ecological transitional zone that extensively covers and forms more than $60 \%$ of the land surface of the study area. The open woodland of the area has diverse structure, as in some places the open woodland exhibits a structure described as tree grass mosaic, a mixture of grass and trees with the trees being the dominant cover (FPP-PASCO-FC Report 2013). This form occurred in the northern part of the study area around Grubago, Wawato and Kparia. These areas are often low population density with agricultural lands that have secondary growth as a result of long fallow periods. Additionally, the study revealed the open woodland expanded consistently over the study period at 0.17 (1990-2010). The rate of expansion was far below the rate of loss of the dense woodland, which was 0.018 . This implies that some of the dense woodlands were being converted to other land use between 2000 and 2010 such as cropland, charcoal burning and grassland. The woodlands expanded by 0.11 ha for ten years at an annual rate of 0.011 ha and between 1990-2010, it expanded by 0.28 ha at an annual rate of 0.01 ha. It is projected that by 2020 , the open woodland will expand at an annual rate of 0.01 ha. In the northern section, there is a heavy presence of Fulani herdsmen, which has caused over grazing and degradation of the open woodland (FPP-PASCO-FC Report 2013).

The small diameter size trees make the area attractive to charcoal producers and firewood collectors. However, these areas have very high potential for restoration, although these are very vulnerable to fire as a result of the high percentage of grass cover. The other form is made of trees with diameters varying from 15 to $30 \mathrm{~cm}$ forming clusters and having stocking varying from 60 to $200 \mathrm{~cm}$ stems/ha (FPP-PASCO-FC Report 2013). The under growth is grass and shrubs making it very susceptible to fires and over grazing. It is worth noting that the entire landscape has the potential of being transformed to grassland if not properly managed, considering the high proliferation of Fulani herdsmen and high demand of wood and wood products by farmers around Bawena and Anyantoo (FPP-PASCO-FC Report 2013). Also, the expansion of agricultural lands due to poor farming practices such as shifting cultivation and fast urbanization as a result of the newly created districts by the Central Government, which triggers expansion in infrastructure development, poses a threat to the productivity of the land and maintenance of vegetation cover. From the analysis, it is observed that the open woodland expanded slowly, while the dense woodland decreased faster.

\section{Riverine forest}

Riverine vegetation and wetlands are recognized worldwide as ecologically endangered ecosystems (Tockner and Stanford 2002). The study area is endowed with numerous streams and rivers and abundant surface water. The riverine forest contains rare and endangered species and prevents the water bodies from drying out, as 
well as acting as bio-filters purifying and enhancing the underground water, which is the main source of drinking water to the community. This study has revealed that the Riverine forest expanded slightly between 1990-2000 by $1,172.1$ ha and loss between 2000-2010 epochs by 1810 ha. For the 20 -year period, it recorded a decline of 367.7 ha between 1990-2010 epochs. The continuous loss of the riverine forest is a result of the loss in dense woodland, farming along water courses and bush burning, leading to siltation of the river systems. The riverine areas have trees with large diameters, well-formed boles and are always green throughout the year. Farmers are by law expected to leave a buffer of $50 \mathrm{~m}$ along water courses to protect the forest along the water bodies, however, this law is seriously violated and farming along the water courses is resulting in heavy siltation of the rivers, thereby reducing the capacity of the river and causing flooding during the rainy season (FPP-PASCO-FC Report 2013).

Farming along the rivers in dry season has necessitated the diversion of water courses for the cultivation of vegetables in dry season and resulted in destruction, disturbance or disruption of the ecological processes within the forest ecosystems and enhancing oxygen-depletion. In addition, this area also serves as the corridor for wildlife, and according to research, the environmentally sensitive areas such as riparian forest, steep hilly, rocky outcrop and swamps are important for the longterm maintenance of biological diversity, soil, water or other natural resources. These are unique and fragile areas that offer refuge and sustenance to a diverse body of terrestrial wild animals, plants and sensitive aquatic organisms (Jordan 2013).

\section{Cropland}

The study has confirmed that agriculture is the main stay of the people in the study area and crops grown are mainly annual such as yam, maize, cassava sesame and beans. The average farm size is about 2 ha and most farmers still rely on hoe, cutlass and fire for tilling the land. Traditionally, trees and cash crops such as cocoa, citrus and oil palm are not grown, although there are few patches of teak, mango and cashew plantations found in the area (FPP-PASCO-FC Report 2013). The results show that cropland expanded by $70 \%$ of its size and $6 \%$ of the total landscape in the 20 -year period. This invariably accounted for the dramatic increase in the open woodlands where the farms are located and corresponding decrease of the dense woodland that is being converted to farms. Farming activities are mainly on subsistence level with few commercial farms, and generally, farming is done haphazardly due to the lack of agricultural extension services and the absence of a Land-use Plan to guide farmers and other stakeholders for prudent utilization of the land and land resources. Agriculture is not based on soil-crop suitability matching but depends solely on decision of the farmer. The presumption by the farmers that productivity is positively correlated to the size of the land invariably results in rampant clearing of more virgin (new) lands because much of the newly cropped land is poorly managed and depletes very fast, thereby forcing the farmer to convert more woodlands to agricultural lands. Engaging agricultural extension officers to carry out education on basic agronomic practices will be very useful in this regard (FPP-PASCO-FC Report 2013).

\section{Built-up}

The study areas fall within the West and North Gonja district of the Northern Region, which is sparsely populated and has the lowest population density in Ghana. It is interesting to note that the towns are however expanding at an alarming rate, as shown in the land use change map. From the result in Table 4, settlements that include road surfaces, gravel pits and human habitation expanded by original size in 1990 and $1 \%$ of the total land surface in the study area. This shows that human settlement is expanding very fast due to urbanization, but the expansion is not commensurate with the vast expanse of land, so the area still remains sparsely populated. The settlements are generally hamlets, small communities and towns that constitute $70 \%$ of the class and few urban settlements. Aside human settlements, the results of the study have revealed the existence of patches of bare surface due to sand winning activities for road construction (FPP-PASCO-FC Report 2013).

\section{Water}

Water include the, perennial rivers, streams and ponds. This class remains generally stable, but varies seasonally. The area is generally flat with iron pans and rocky outcrops in some areas. The underlying rocks are impervious to water; therefore, rain water tends to remain on the surface resulting in intermittent flooding. Pools 
of water are collected in the low-lying areas in the rainy season and dries up in the dry season. The main source of drinking water is boreholes, which were found in most villages (FPP-PASCO-FC Report 2013).

\section{Drivers of Deforestation and Forest Degradations}

Indufor Oy (2015) identifies the key drivers of deforestation in Ghana and these drivers were confirmed during the field visits and community interaction in the study area. Drivers identified during the study include: slash and burn farming practices, wood harvesting, bush burning and grazing. According to Brown et al (1998), in order to ensure global food security and avert dangerous climate changes, the world faces the pressing dual challenge of protecting its remaining natural forests and enhancing food production in a sustainable and resilient manner (FPP-PASCO-FC Report 2013). These drivers are elaborated in the below-mentioned factors:

\section{Annual Wildfires}

The site visits and oral interviews of the people of the study area revealed that annual fires in the Mole landscape suppress all woody vegetation. However, low intensity fires that occur in the early part of the dry season had little effect on stem biomass and mortality, even when they occur frequently than high intensity fires lead to high top-kill rate. The base of trees is normally not killed, which is in contrast to fire as a result of farming that normally kills the base of the stem. The base of the tree that is not killed will sprout soon after the fire is over, that is, in the pre-season greening-up, which is a dominant phenomenon of savanna ecosystem (Ryan et al. 2011). The regenerated stems have become very vulnerable to fires in the ensuing years resulting in the transformation of the woodland to shrub land, which reduces the carbon content of the landscape. Furley et al. (2009) stated that long term exclusion of fire leads to the formation of closed canopies and a succession towards forest.

The reduced tree cover of savannas allows enough light down to the ground to facilitate the growth of large quantities of grass, which provide food for the wildlife and also serve as fuel for the widespread fires that affect the woody biomass and the carbon. Burnt areas had very little carbon and juvenile trees, and showed succession from woodlands to shrub land. Manipulat- ing fire intensity rather than frequency seems to be the most practical approach to limiting degradation by fire in the Mole landscape. Hence, the need for promotion of early burning regimes to avoid the damaging intensity when there is build-up of combustible material in the dry season (FPP-PASCO-FC Report 2013).

\section{Extensive Farming}

Farming in the northern part of Ghana and the study area in particular has been through slash and burn and sifting cultivation. These practices require using fire to prepare the land for cultivation. Fires are often left uncontrolled and get out of hand destroying the vegetation, thereby causing soil depletion as a result of erosion and deposition of ash, which eventually turn to Potash, thus causing the soil $\mathrm{pH}$ to rise. In addition, it burns the tracer elements into the atmospheres and kills wildlife and other microorganisms needed for plant growth. The study has revealed that the main crops grown are food crops and the yields are falling due to the fast depletion of soil fertility as a result of burning, which compels them to shift their farms to new areas of high fertility. This results in clearing vegetation and causing loss of carbon stocks as well as emission of carbon dioxide to the atmosphere (FPP-PASCO-FC Report 2013).

Farmers deliberately set wildfire to the base of the trees thereby killing them to increase yield in the immediate seasons as they perceive the trees to be casting shade and over competing with food crops for soil nutrients, consequently suppressing the growth of crops and reducing the yield. However, these yields are not sustainable because the soil becomes vulnerable to erosion and alkaline as a result of deposition of ash (FPPPASCO-FC Report 2013).

\section{Wood Harvesting}

The study also revealed massive timber harvesting in the dense woodland and along the riverine forest. The activity has been so intensive and extensive that every spot within the study area visited; there was some form of timber activity. This is a very worrying situation for resource management in the area. Savannah woodland, by nature, grows very solely and the trees have large crowns and profuse branching. The structure and form of the trees suppress the growth of weeds and maintain the natural state of the woodland. When one or two trees are removed from the same 
area, it opens the canopy, which results in grass colonizing the area that adds up to fuel loads and thereby aggravate the fire intensity. The fires burn away the undercover, scorch the standing trees and reduce their lifespan (Werner and Prior 2013). In most instances, the burnt trees do not survive to the next season. They die off and are captured as dead standing during the next accounting season. This aggravates the fire situation, reduces the carbon stocks as well as releases $\mathrm{CO}_{2}$ to the atmosphere.

\section{Grazing}

Fulani herdsmen to induce fresh growth of grass for the cattle. The fires are left and uncontrolled and they burn beyond their intended regions. Resulting in the destruction of vegetation, food crops and sometimes human life. In the carbon analysis, it was revealed that the natural woodland that suffered from no burning had more tally trees (i.e., live standing trees), high numbers of juveniles (i.e., potential for natural regeneration), and few under growth of grass. Such plots yielded a high amount of above ground biomass and carbon. The previously burnt areas had higher number of dead standing and dead down trees, but very few standing trees and no juveniles. Such areas were transitioning from dense to open woodlands and to grass, and therefore, had little biomass and carbon (FPP-PASCO-FC Report 2013). Freshly burnt areas had huge deposition of ash and no dead down tress, which meant that they had been consumed by fires, scorched standing trees that may not survive the next cycle. Such areas also registered lesser biomass and carbon.

\section{Conclusions}

- The deforestation rate between 1990 and 2010 was $0.11 \%$. This was calculated to be 676 ha per annum. The riverine and the dense woodland diminished faster, contributing to the expansions of open woodlands, cropland grassland and settlement.

- The study area has immense potential of carbon sequestration, as the average value was $80 \mathrm{t} / \mathrm{ha}$. The dense woodland recorded the highest carbon storage of $194 \mathrm{t} / \mathrm{ha}$ and the lowest from the grasslands of $7 \mathrm{t} / \mathrm{ha}$. However, the annual bushfires and unsustainable agricultural practices have serious consequences of the carbon sequestration potential of the area.

- Burnt areas affected the various carbon pools in diverse ways, for example, freshly burnt areas had no juvenile trees, dead down branches nor dead standing. However, previously burnt areas showed dead down branches or dead standing and few juvenile trees.

- The study has revealed that the main drivers of deforestation in the area are the annual bush fires, unsustainable agricultural activities, grazing, charcoal production and, in recent times, unsustainable harvesting of wood for timber and fuelwood.

- The woodlands have diverse structure and form, and hold substantial amount of carbon. However, the woodlands are fragile and vulnerable to human intervention and annual bush fires. The transformation of the woodland is facilitated by fires, farming and logging. It goes through dense to open to shrub and to grassland. The open woodland and the shrub phase are the most fragile because of the high percentage grass cover that serves as fuel for the annual bush fires. Bush burning affect the diversity, structure, form and the carbon sequestration potential of the woodlands and releases carbon dioxide into the atmosphere. Burning affect soil fertility and productivity, and consequently, crop yields increase at the expense of the woodlands that are being converted to croplands.

- Agriculture is still rudimentary and remains one of the drivers of land conversion.

\section{Acknowledgement}

Much appreciation to Mr. Yakubu Mohammed and Mr. Emmanuel Donkor (RMSC - Forestry Commission, Ghana), Rocha Ghana and IUCN for the support.

\section{References}

Antwi E.K., Owusu-Banahene W., Boakye-Danquah J., Mensah R., Tetteh J.D., Nagao M., Takeuchi K. 2017. Sustainability assessment of mine-affected communities in Ghana: towards ecosystems and livelihood restoration. Sustainability Science, 1-21. 
Asante W. 2014. Operational Guidance and Standards for National and Subnational REED+ Programs in Ghana. Forestry Commission of Ghana.

Ayuk E.T., Oku E.E. 2017. Promoting Green Economy: Implications for Natural Resources Development, Food Security and Poverty Reduction in Africa. United Nations University Institute for Natural Resource.

Brown S., Lugo A.E., Gillespie A.J.R. 1998. Biomass estimation methods for Tropical Forests with applications to Forest inventory data. Forest Science, 35 (4), 381-902.

Forestry Commission of Ghana. 2014. SOP - 003 Acquisition of Remote Sensing Data and Generation of Spatial Activity Data. https://fcghana.org/nrs/ index.php/.

FPP-PASCO-FC Report. 2013. Mapping of Forest Cover and Carbon Stock in Ghana - Pilot study which covered the SECOND and THIRD TIERS recommended in the Good Practice Guidelines of the Intergovernmental Panel on Climate Change (IPCCGPG) for REDD+ planning.

Furley P.A., Rees R.M., Ryan C.M., Saiz G. 2009. Savanna burning and the assessment of long-term fire experiments with particular reference to Zimbabwe. Progress in Physical Geography in press.

GSS. 2013. 2010 Population and Housing Census National Analytical Report. Accra, Ghana.

Haberl H., Mbow C., Deng X., Irwin E.G., Kerr S., Kuemmerle T., Mertz O., Meyfroidt P., Turner I.I.B.L. 2014. Finite land resources and competition. In: Rethinking Global Land Use in an Urban Era (eds.: K.Seto, A. Reenberg). MIT Press, Cambridge.

Houghton R.A. 2005. Aboveground forest biomass and the global carbon balance. Global Change Historical Deforestation Baselines in Support of UNFCCC REDD. Biology, 11, 945-958.

Indufor Oy. 2015. Development of Reference Emissions Levels and Measurement, Reporting and Verification System in Ghana FC/FCPF/MRV/REL/ RFP/01/2013.
Innes J.L. 2016. Forests and carbon. Sustainable Forest Management: From Concept to Practice, 189.

IPCC. 2003. Good Practice Guidance for Land Use, Land-Use Change and Forestry.

IPCC. 2007. Climate Change 2007: The Physical Science Basis. Cambridge University Press, Cambridge, United Kingdom and New York, NY, USA.

Jordan C.F. 2013. A History of Unsustainability in Agriculture. In: An Ecosystem Approach to Sustainable Agriculture (ed.: C.F. Jordan). Springer Netherlands, 39-62.

Lillesand T., Kiefer R. 2008. Remote sensing and image interpretation. JohnWiley \& Sons Inc., New York.

Mackay R. 2014. The atlas of endangered species. Routledge.

Rockström J., Steffen W., Noone K., Persson Å., Chapin F.S., Lambin III, E.F., Lenton T.M., Scheffer M., Folke C., Schellnhuber H.J., Nykvist B., de Wit C.A., Hughes T., van der Leeuw S., Rodhe H., Sörlin S., Snyder P.K., Costanza R., Svedin U., Falkenmark M., Karlberg L., Corell R.W., Fabry V.J., Hansen J., Walker B., Liverman D., Richardson K., Crutzen P., Foley J.A. 2009. A safe operating space for humanity. Nature, 461, 472-475, doi: 10.1038/461472a.

Ryan C.M., Williams M., Grace J. 2011. Above and belowground carbon stocks in a Miombo woodland landscape of Mozambique. Biotropica, 43, 423-432.

Srivastav A., Srivastav S. 2015. Ecological Meltdown: impact of unchecked human growth on the earth's natural systems. The Energy and Resources Institute (TERI).

Tockner K., Stanford J.A. 2002. Riverine flood plains: present state and future trends. Environmental Conservation, 29 (3), 308-330.

Van DerWerf G.R., Morton D.C., Defries R.S. 2009. $\mathrm{CO}_{2}$ emissions from forest loss. Nature Geoscience, 2, 737-738.

Werner P.A., Prior L.D. 2013. Demography and growth of subadult savanna trees: interactions of life history, size, fire season, and grassy understory. Ecological Monographs, 83 (1), 67-93. 